\title{
KIAA1522 potentiates TNFa-NFKB signaling to antagonize platinum-based chemotherapy in lung adenocarcinoma
}

\author{
Boshi Wang ${ }^{1 * \dagger} \mathbb{D}$, Tiantian Jing ${ }^{1 \dagger}$, Weilin $\mathrm{Jin}^{2}$, Jinnan Chen ${ }^{3}$, Chengsi Wu ${ }^{1}$, Mingrong Wang ${ }^{4}$ and Yizhen Liu ${ }^{5,6^{*}}$
}

\begin{abstract}
Background: The platinum-based chemotherapy is the first-line regimen for the treatment of Non-small cell lung cancer (NSCLC). However, the therapeutic efficiency is largely limited by tenacious chemo-insensitivity that results in inferior prognosis in a cohort of patients. It has been known that KIAA1522 is aberrantly expressed and implicated in several types of solid tumors including NSCLC. Nowadays, knowledge about this gene is quite limited. Here, we aimed to identify the role of KIAA1522 in lung adenocarcinomas, and the molecular events that underlie KIAA1522mediated chemoresistance to the platinum.

Methods: Immunohistochemistry were used to detect KIAA1522 expression in clinical NSCLC samples. Then, the survival analyses were performed to assess the link between KIAA1522 expression and overall survival or therapeutic outcome. In vivo depletion of KIAA1522 in adenocarcinoma cells were achieved by adeno-associated virusmediated sgRNA/Cre delivery into the conditional $\mathrm{Kras}^{\mathrm{G} 12 \mathrm{D}} / \mathrm{Cas} 9$ expressed mice, which were designated to identify the roles of KIAA1522 in tumorigenesis and/or chemotherapy responses. The effects of KIAA1522 and downstream molecular events were studied by pharmacology in mice model and assays using in vitro cultured cells. The clinical relevance of our findings was examined by data-mining of online datasets from multiple cohorts.

Results: The clinical evidences reveal that KIAA1522 independently predicts both the overall survival and the outcome of platinum-based chemotherapy in lung adenocarcinomas. By using a Kras ${ }^{G 12 D}$-driven murine lung adenocarcinoma model and performing in vitro assays, we demonstrated that KIAA1522 is a critical positive regulator of lung adenocarcinoma and a modulator of cisplatin response. KIAA1522 potentiates the TNFa-TNFR2NFKB signaling which in turn intensifies recalcitrance to cisplatin treatment. These results were further manifested by integrative bioinformatic analyses of independent datasets, in which KIAA1522 is tightly associated with the activity of TNFa-NFkB pathway and the cisplatin-resistant gene signatures. More strikingly, overexpression of KIAA1522 counteracts the cisplatin-induced tumor growth arrest in vivo, and this effect can be remarkably diminished by the disruption of NFKB activity.

(Continued on next page)
\end{abstract}

\footnotetext{
* Correspondence: wbs137@shsci.org; aliuyz@126.com

†Boshi Wang and Tiantian Jing contributed equally to this work.

${ }^{1}$ State Key Laboratory of Oncogenes and Related Genes, Shanghai Cancer

Institute, Renji Hospital, Shanghai Jiao Tong University School of Medicine,

Shanghai 200032, China

${ }^{5}$ Department of Medical Oncology, Fudan University Shanghai Cancer

Center, Shanghai 200032, China

Full list of author information is available at the end of the article
}

(c) The Author(s). 2020 Open Access This article is licensed under a Creative Commons Attribution 4.0 International License, which permits use, sharing, adaptation, distribution and reproduction in any medium or format, as long as you give appropriate credit to the original author(s) and the source, provide a link to the Creative Commons licence, and indicate if changes were made. The images or other third party material in this article are included in the article's Creative Commons licence, unless indicated otherwise in a credit line to the material. If material is not included in the article's Creative Commons licence and your intended use is not permitted by statutory regulation or exceeds the permitted use, you will need to obtain permission directly from the copyright holder. To view a copy of this licence, visit http://creativecommons.org/licenses/by/4.0/ The Creative Commons Public Domain Dedication waiver (http://creativecommons.org/publicdomain/zero/1.0/) applies to the data made available in this article, unless otherwise stated in a credit line to the data. 


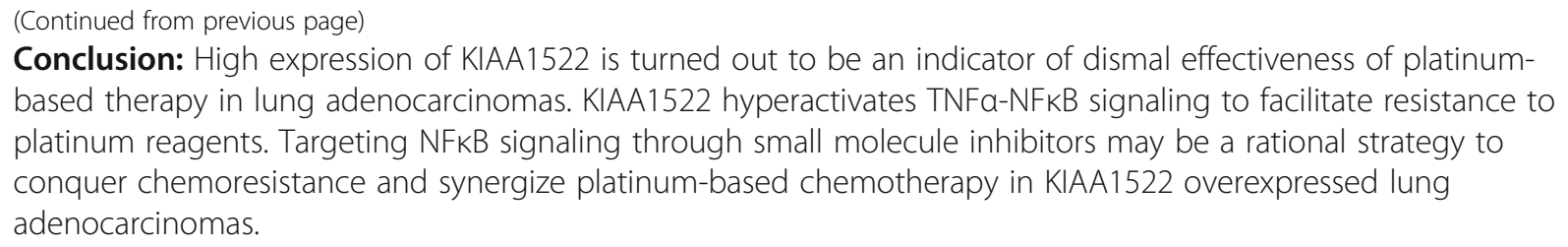

Keywords: KIAA1522, Lung adenocarcinoma, NFkB, Chemoresistance, Platinum-based chemotherapy

\section{Background}

Lung cancer is the most common type of human malignancy and causes more cancer-related mortality than other diseases worldwide [1]. Non-small cell lung cancer (NSCLC) is the major histological subtypes of lung cancer, which is comprised by lung adenocarcinoma (ADC) and lung squamous cell carcinoma (SCC) [2]. Several therapeutic advances have been achieved in recent years, especially the progress in target therapy and the emergence of immunotherapy [3, 4]. However, a limited number of subtypes are benefited from these two methods and the overall survival rates of NSCLC remain low. Curative surgery is the preferred choice for the majority NSCLC patients in early and late stage. Unfortunately, even the NSCLC patients in the advanced stage underwent complete resection, about $70 \%$ patients have dismal prognosis due to tenacious drug resistance $[2,5]$. Nowadays, platinum-based chemotherapy remains the cornerstone of routine adjuvant chemotherapy. This approach moderately improves 5-year survival rate, whereas its long-term clinical effectiveness severely impeded by the inherited or acquired resistance to the platinum-based reagents [6-8].

Cisplatin is one of the most widely used and studied platinum-based cytotoxic drugs. But none of specific biomarker that guide cisplatin usage in the clinical practice. Also, there is still lack of effective reagents overcoming cisplatin-resistance. It has been known that some genetic variations and alterations of key signaling pathways can enhance cisplatin insensitivity [9]. NFKB signaling is one of the crucial signaling pathways associated with chemoresistance [10-12]. Hyperactivation of $\mathrm{NF} \mathrm{B}$ signaling is implicated in multiple types of cancers, contributing to tumor initiation, development, progression and responses to extracellular stimulations [13]. The transcription factor $\mathrm{NF}_{\mathrm{K}} \mathrm{B}$ is a pleiotropic hetero- or homo-dimer, complexed by RelA/p65, RelB, p52, p50 or c-Rel. The pro-inflammatory cytokine TNFo is a robust upstream regulator of $\mathrm{NF} \times \mathrm{B}$. Once stimulated by $\mathrm{TNF} \alpha$, the TNF receptors recruit certain cytoplasmic proteins to form an IKK regulating complex to active IKK $\alpha / \beta$, then IKK $\alpha / \beta$ phosphorylates IKB and unleashes p50: p65 complex into nucleus. The activation of NFKB transcriptional factor regulates the expression of certain anti- apoptotic genes, such as XIAP, BCL-2 and BIRC5/survivin, which counteract the cytotoxic effects of cisplatin [14-16]. Notably, there is ample evidence supporting that $\mathrm{NFKB}$ is essential for the development and chemoresistance of lung adenocarcinoma [17-20].

The functions of KIAA1522 was not investigated until the recent years. It was reported as an early-diagnostic biomarker in one of our previous work [21]. Furthermore, we found that KIAA1522 promotes NSCLC development via the RAS-MEK-ERK pathway [22]. Otherwise, KIAA1522 accelerates the metastatic ability of esophageal carcinoma cells and breast cancer cells $[23,24]$. Here, we describe an unappreciated role of KIAA1522 in potentiating TNF $\alpha-\mathrm{NF} K \mathrm{~B}$ signaling, and thereby give rise to cisplatin resistance. Also, we will explore several research questions: whether KIAA1522 contributes to chemoresistance in experimental lung cancer models. What are the potential mechanisms responsible for KIAA1522-induced chemoresistance? And how can we restore sensitivity to platinum-based therapy in KIAA1522 high expressed ADC samples. These efforts may suggest a future avenue for patients' treatment.

\section{Methods}

\section{Patients and samples}

The NSCLC samples were procured in the Cancer Hospital, Chinese Academy of Medical Sciences and Peking Union Medical College (CAMS \& PUMC). Primary tumor tissues and adjacent non-tumoral lung tissues were excised and pathological diagnoses by experienced pathologists. The tissue samples were fixed with neutral buffered formalin ( $\mathrm{pH}$ 7.4) and paraffin-embedded for the construction of tissue microarrays. All of the tissues were residual specimens after diagnostic sampling. None of the patients received pre-surgical treatment. The basic clinicopathologic data were listed in Table S1. This study was approved by the Ethics Committee/ Institutional Review Board of the Cancer Institute (Hospital), PUMC/CAMS (No. 12-098/632). Written informed consent forms were obtained from patients for sampling and research. And all the methods in our study were carried out in accordance with the approved guidelines. 


\section{Cell culture}

The human lung adenocarcinoma cell lines A549 and NCI-H1299 were acquired from the American Type Culture Collection (ATCC, Manassas VA, USA). The murine lung cancer cell line 889-DTC (889) was a kindly provided by professor Winslow $[25,26]$. HEK293T cells were acquired from the American Type Culture Collection (ATCC, Manassas, VA, USA). Cell lines were maintained at $37^{\circ} \mathrm{C}$ in $5 \% \mathrm{CO} 2$ in Dulbecco's modified Eagle medium supplemented with $10 \%$ fetal bovine serum.

\section{Antibodies and reagents}

Recombinant human TNF $\alpha$ (AF-300-01A) was purchased from PeproTech. Cisplatin (HY-17394) was from MedChemExpress, QNZ (S4902) and Cycloheximide (CHX) was from Selleck. The primary antibodies used in this work are as follow: KIAA1522 (WB, 1:1000; IHC, 1: 200; HPA032050, Sigma ImmunoChemicals, St Louis, MO, USA), Phospho-NF-kB p65 (Ser536) (WB, 1:1000; CST, 3033); NF-kB p65 (WB, 1:1000; CST, 8242),

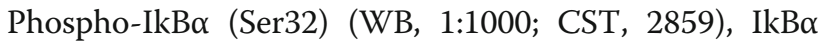
(WB, 1:1000; CST, 4814), Phospho-IKK $\alpha / \beta$ (Ser176/180) (WB, 1:1000; IHC, 1:150; CST, 2697), IKK $\alpha$ (WB, 1: 1000, CST, 11930), TNFR1 (WB, 1:1000, Proteintech, 21, 574-1-AP), TNFR2 (WB, 1:1000; IHC, 1:50, Proteintech, 19,272-1-AP), GAPDH (WB, 1:1000, Santa Cruz, sc-25, 778), $\beta$-actin (WB, 1:2000, Santa Cruz, sc-47,778), Ki67 (IHC, 1:200, GeneTex, GTX16667), cleaved caspase-3 (IHC, 1: 500, CST, 9664).

\section{Plasmid and lentivirus package}

C-terminal Flag-tagged KIAA1522 were cloned into pLVX-IRES-ZsGreen plasmid. The forward shRNA sequences for targeting human KIAA1522 are as follow: sh-1: CCGGCCACGGCCTTCGATACTTATGCTCGA GCATAAGTATCGAAGGCCGTGGTTTTTG; sh-2: CCGGCCTACCTGTCGAAGTTGATTCCTCGAGGAA TCAACTTCGACAGGTAGGTTTTTG. shRNA sequences were cloned into pLKO.1 plasmid (Sigma-Aldrich, Missouri, USA). The sgRNAs for targeting mouse kiaa1522 gene are as follow: sg-1: GCCGAGAGTG ACAACCGTCA; sg-2: AGTGGGAGACCTCCTCAT CT. The sgRNA sequences were cloned into lentiCRISPRv2 plasmid which was a gift from Feng Zhang (Addgene plasmid \# 52961) [27] The lentiCRISPR-EGFP sgRNA1 plasmid (Addgene plasmid \#51760) from Feng Zhang was used as sgRNA control [28]. HEK293t cells were used for lentivirus packaging. Lentiviral vectors expressing target genes were co-transfected with lentiviral packaging plasmids psPAX2 (Addgene plasmid, 12,260) and pMD2.G (Addgene plasmid, 12,259) with Lipofectamine 3000 (Invitrogen). The medium containing lentivirus was harvested at $48 \mathrm{~h}$ and $72 \mathrm{~h}$ after transfection and used to infect cultured cell lines. Transduced cells were isolated by puromycin selection or FASC sorting.

\section{Adeno-associated virus (AAV) production and mice management}

The sgRNA sequences for AAV-mediated in vivo kiaa1522 editing are GGAAGTCAGGAAGGCGACGG and GCCGAGAGTGACAACCGTCA. Each sgRNA was connected next to a U6 promoter and serially cloned into the pAAV-U6-gRNA v2.0-CMV-NLS-Cre-3FLAGP2A-EGFP-WPRE vector (OBiO Technology (Shanghai) Corp., Ltd), which express a nuclear localization signal fused Cre protein. The sgkiaa1522-expressing AAV was generated by co-transfection of HEK293T cells with AAV expression vector, AAV helper plasmid and AAV Rep/Cap plasmid. The cells were lysed by a freeze-thaw procedure at $72 \mathrm{~h}$ after transfection. The viral particles were purified by iodixanol step-gradient ultracentrifugation and concentrated by a molecular-mass-cutoff ultrafiltration device. a non-sense sequence GCACTACCAG AGCTAACTCA was also cloned into the PAAV vector to generated the sg-control virus. The B6-Gt (ROSA)26-

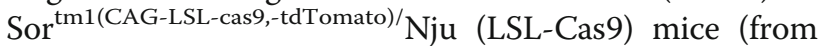
Nanjing University Model Animal Resource Platform) were crossed by $\operatorname{Kras}^{(L S L-G 12 D)}$ mice (from Shanghai Model Organisms Center, Inc.) to generate the conditionally Cas9/ Kras ${ }^{\mathrm{G} 12 \mathrm{D}}$ expressing mice. 8-12-week-old mice were anesthetized by intraperitoneally injection of pentobarbital sodium, and then delivered intratracheally by the recombinant AAV particles $\left(2 \times 10^{11} /\right.$ mouse $)$. For in vivo cisplatin treatment, the mice were treated intraperitoneally by cisplatin $(3.5 \mathrm{mg} / \mathrm{kg}$ B.W.) once a week for one month.

\section{Allograft/xenograft tumor model and in vivo pharmacology}

For in vivo tumorigenesis assays, $5 \times 10^{5} 889$ cells were subcutaneously injected into C57BL/6 mice (5-6 weeks age) for 1 month. $5 \times 10^{6}$ A549 cells were subcutaneously injected into BALB/c nude mice (5-6 weeks age) for 2 months. The control and KIAA1522-overexpressed A549 cells were subcutaneously injected into C57BL/6 mice. Two weeks later, the mice were subjected to drug treatment: cisplatin $(7 \mathrm{mg} / \mathrm{kg}$ B.W.) once a week and QNZ (1 mg/kg B.W.) twice a week via intraperitoneal injection. The tumor volumes were measured by the formula: $\left(\right.$ length $\times$ width $\left.^{2}\right) / 2$.

\section{Colony formation assays}

To assess the inhibitory effects of small-molecular reagents in vitro, $1 \times 10^{4}$ cells were seeded in 12-well plates. $72 \mathrm{~h}$ later the cells were treated with vehicle, cisplatin $(10-20 \mu \mathrm{M})$, QNZ (500 $\mathrm{nM})$, or combination for 
$48 \mathrm{~h}$. Then the cells were fixed by methanol and stained with crystal violet (Beyotime, C0121).

\section{Cell viability analysis}

Cells were seeded at 2000 cells in $200 \mu \mathrm{L}$ DMEM per well in 96-well culture plates. At the indicated time points, $20 \mu \mathrm{l}$ Cell Counting Kit- 8 reagent (Beyotime, C0039) was added to each well and incubated at $37^{\circ} \mathrm{C}$ for $2-4 \mathrm{~h}$. The absorbance values (OD $450 \mathrm{~nm}$ ) were measured using a spectrophotometer. The absorbance values at $600 \mathrm{~nm}$ were used as references.

\section{Luciferase reporter assay}

889 cells with or without TNF $\alpha(10 \mathrm{ng} / \mathrm{ml})$ treatment were transiently transfected with $\mathrm{NF \kappa B}$ luciferase reporter plasmid $(1 \mu \mathrm{g})$, and $20 \mathrm{ng}$ of the Renilla luciferase plasmids. $48 \mathrm{~h}$ post-transfection, the firefly and Renilla luciferase activities were monitored using the Dual-Luciferase Reporter Assay System (Promega). $\mathrm{NFKB}$ signaling activity is determined by the ratio of firefly to Renilla luciferase activity.

\section{Western blot and co-immunoprecipitation}

Cells were lysed by RIPA buffer (Thermo Fisher Scientific, 89,901) with protease inhibitors cocktail (Roche Diagnostics, 05892970001) and phosphatase inhibitor cocktail (Roche Diagnostics, 04906845001). The lysates were clarified by centrifugation at $13000 \mathrm{~g}$ for $30 \mathrm{~min}$ at $4{ }^{\circ} \mathrm{C}$. Protein concentrations were determined by $\mathrm{BCA}$ protein assay kit (Thermo Fisher Scientific, 23,225) followed by boiled with loading buffer. Protein samples $(50-150 \mu \mathrm{g})$ were separated through SDS-PAGE, then transferred to nitrocellulose filter membrane (Pall Corporation) blocked and incubated with the primary antibodies. After washing with TBST, the blots were incubated with Anti-rabbit IgG HRP-linked antibody (CST, 7074) and Anti-mouse IgG HRP-linked antibody (CST, 7076), then visualized by the SuperSignal West Dura Extended Duration Substrate (Thermo Fisher Scientific, 34,076).

Co-immunoprecipitation (Co-IP) was performed using Protein G-agarose suspension (Millipore, 16-266). The cells were lysed by IP lysis buffer (Beyotime Institute of Biotechnology, P0013), and then incubated by $50 \mu \mathrm{l}$ of Protein G-agarose suspension for $3 \mathrm{~h}$ at $4{ }^{\circ} \mathrm{C}$ on a rocking platform to reduce non-specific binding. After removing the beads, the supernatant was supplemented with the primary antibodies followed by incubation for another $3 \mathrm{~h}$ at $4{ }^{\circ} \mathrm{C}$. A total of $100 \mu \mathrm{l}$ of Protein G-agarose was then mixed to each sample, and the incubation was continued overnight on a rocking platform. The immunoprecipitates were collected by centrifugation and washed three times with the TBS. The agarose was boiled with loading buffer and subjected to western blot analysis.

\section{Immunohistochemistry}

The tissue microarrays and slides were deparaffinized, rehydrated, immersed in 3\% hydrogen peroxide solution for $15 \mathrm{~min}$, heated in citrate buffer ( $\mathrm{pH}$ 6.0) for $25 \mathrm{~min}$ at $95^{\circ} \mathrm{C}$, and cooled for at least $60 \mathrm{~min}$ at room temperature. Between each incubation step, the slides were washed three times with PBS (pH 7.4). After blocked with $10 \%$ normal goat serum for $30 \mathrm{~min}$ at $37^{\circ} \mathrm{C}$ and washed, the slides were incubated overnight at $4{ }^{\circ} \mathrm{C}$ with primary antibodies against target proteins and visualized using the PV-9000 Polymer Detection System (GBI, USA) following the manufacturer's instructions or GTVisionTMIII Detection System/Mo\&Rb (GeneTech, GK500710). After washing with PBS, the slides were counterstained with hematoxylin.

\section{Immunohistochemical assessment and analysis}

Protein expression levels were determined on the basis of staining intensity and the percentage of immunoreactive cells. Staining intensity was rated as 0 (negative), 1 (weakly positive), 2 (moderately positive), and 3 (strongly positive). The percentage of immunoreactive cells was graded as $0(0 \%), 0.5(1-10 \%), 1(11-20 \%), 2$ (21-50\%), $3(51-80 \%)$, or $4(81-100 \%)$. The average of tumor cell staining intensity score multiplied by the percentage of positive cells score represented the final score of one sample. The prognostic value of certain protein was evaluated by univariate or multivariate cox regression analyses in different subtypes of patients using $\mathrm{R}$ packages survival and survminer. The Nomogram survival predictive model was constructed using RMS package. The optimal cutoff value of IHC staining scores were estimated by $\mathrm{R}$ package maxstat.

\section{RNA sequencing}

Total RNA was extracted by TRIZOL Reagent (Life technologies, CA, USA) according to the manufacturer's instructions, then checked for RIN numbers to inspect RNA integrity by Agilent Bioanalyzer 2100 (Agilent technologies, CA, USA). Qualified total RNA was further purified using RNAClean XP Kit (Beckman Coulter, Inc. CA, USA) and RNase-Free DNase Set (QIAGEN, GmBH, Germany).

Library construction and sequencing were performed at the Shanghai Biotechnology Corp. RNA libraries were prepared for sequencing using VAHTS Universal V6 RNA-seq Library Prep Kit for Illumina (Vazyme, Nanjing, China) before submitted to Illumina Hiseq 2500 system. Clean data was generated by trimming the adaptor and filtering rRNAs using Seqtk (https:/github. $\mathrm{com} / \mathrm{lh} 3 /$ seqtk). Then the clean reads were mapped to 
mouse reference genome (GRCm38) with Hisat2 (version: 2.0.4) to generate the BAM files for each sample. The uniquely mapped fragments of genes were counted by Stringtie (version:1.3.0). Gene expression was evaluated by normalized Fragments Per Kilobase of exon model per Million mapped reads (FPKM) using TMM (trimmed mean of $\mathrm{M}$ values) methods. The raw data of RNA sequencing were deposited in Gene Expression Omnibus (GEO accession number: GSE146072).

\section{Bioinformatic analyses of RNA sequencing results}

The bioinformatic analyses were completed through $\mathrm{R}$ programming language (version 3.6.2) in RStudio software. In the processed RNA-seq data, the genes with more than 500 total counts were subjected to the edgeR analysis to estimate the fold change of each gene between groups. Under the criterion that FDR qvalue $\leq 0.05$, and $\log 2 \mathrm{FC} \geq 1$ or $\leq-1$. The pathway enrichment analysis and the gene-sets enrichment analysis (GSEA) were performed using ClusterProfiler package [29]. The gene expression matrix was transformed to an enrichment score matrix by GSVA package [30] for the comparison of different genesets between groups. The genes significantly down-regulated by KIAA1522depletion in 889 cells were defined as the KIAA1522 positive regulated genes which were constructed to be a geneset by GSEABase package and subjected to the following data mining analyses.

\section{Integrative transcriptome analyses of multi-central datasets}

We downloaded FPKM-normalized RNA-seq data of TCGA-LUAD and TCGA-LUSC datasets together with the associated sample information using TCGAbiolinks package [31]. The FPKM values were transformed to TPM values for the following calculation. The microarray-derived transcriptome datasets from GEO database were downloaded by GEOmirror package and the sample information were acquired by GEOquery package. The lung datasets include GSE3141, GSE8894, GSE13213, GSE11969, GSE37745, GSE31210, GSE30219, GSE50081, GSE43580 and GSE14814. For the GEO datasets including more than one histological type, the lung adenocarcinoma samples were selected for further usage. The single cell sequencing dataset EMTAB-6149 [32] was downloaded from the ArrayExpress website. The data was processed by Seurat package [33] for tSNE dimension reduction and clustering. GSVA program was used to determine KIAA1522 signature score and the scores of genesets associated with $\mathrm{TNF} \alpha-\mathrm{NF}_{\kappa} \mathrm{B}$ signaling and cisplatin resistance. Correlation analyses were performed by cor.test function, and visualized by ggpubr package. Datasets containing survival information were used to perform survival analyses and cox regression analyses by survival and survminer package. Cutoff values for the tested factors were estimated by maxstat package. Meta-analyses were performed using metafor package in the fixed-effects model and the visualization was achieved by either forestplot or ggplot2 package. Alluvial diagram was drawn by alluvial package.

\section{Statistical analysis}

Statistical analyses were conducted by GraphPad Prism 8.0 software and $\mathrm{R}$ statistical packages version 3.6.2. Significant differences between groups were examined through student's t-test. The Kaplan-Meier curves were tested by Log-rank test. All $P$ values $<0.05$ were considered significant.

\section{Results}

\section{High expression of KIAA1522 indicates resistance to} platinum-based chemotherapy in lung adenocarcinoma

Platinum-based adjuvant chemotherapy is the mainstay of post-surgery management for non-small cell lung carcinoma (NSCLC). However, a group of patients experienced drug insensitivity. To identify key genes driving and predicting chemoresistance. We initially screened our previously identified candidate NSCLC biomarkers $[21,34]$ for their prognostic value in the patients receiving platinum-based chemotherapy. After preliminary immunochemical assays in a few paired NSCLC and non-tumoral tissues, there were twenty-two candidate expression-altered proteins that were tested in a total of 598 primary NSCLC samples plus 500 adjacent nontumoral lung tissues dissected from 598 patients, showing extremely high expression of these proteins in tumoral specimens (Supplementary Fig. 1A). Univariate cox analyses were used to examine their association with chemotherapeutic outcome, illustrating that the predictive effectiveness of KIAA1522 were more predominant than other proteins (Fig. 1a). Multivariate cox analysis demonstrated that KIAA1522 was an independent prognostic factor in NSCLC, in either patients receiving chemotherapy or all NSCLC patients (Supplementary Fig. 1B). Similarly, in a nomogram predictive model of NSCLC patients, expression levels of KIAA1522 contributed to lower survival rates (Supplementary Fig. 1C-D).

Immunohistochemistry assays in paired tumoral and non-tumoral tissues showed that KIAA1522 was significantly elevated in both adenocarcinoma and squamous cell carcinoma (Fig. 1b-c). To distinguish the chemosensitive predictive roles of KIAA1522 in different histological subtypes. We performed multivariate cox analyses in adenocarcinoma and squamous cell carcinoma separately. The results revealed that KIAA1522 displayed significant effect in adenocarcinoma patients but not in squamous cell carcinoma (Fig. 1d). In our cohort 


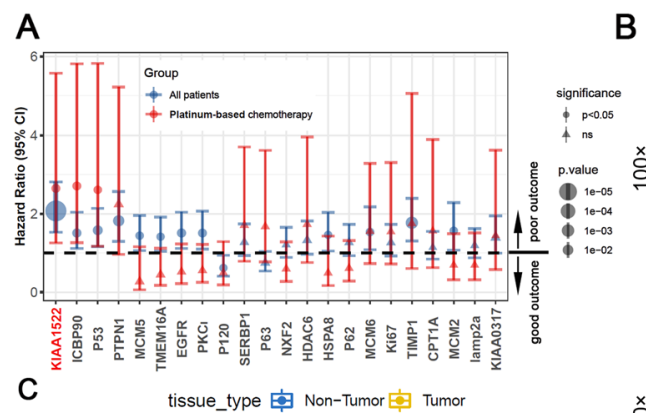

B Non-Tumoral Lung
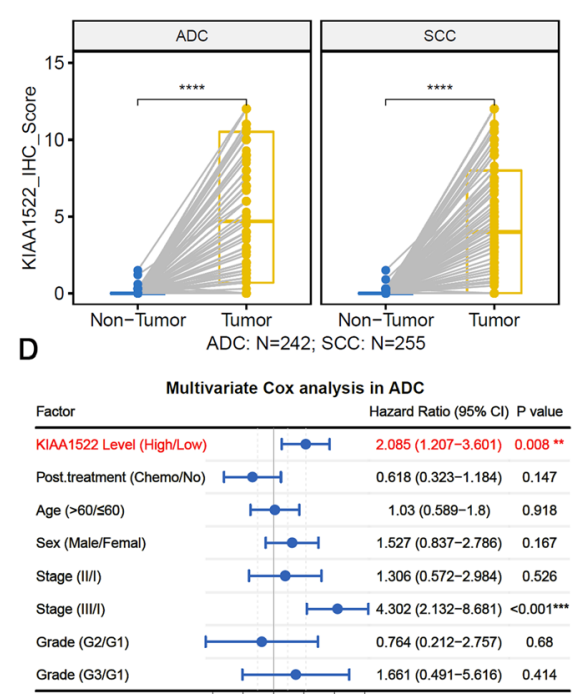

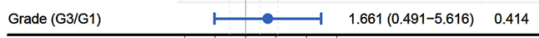

Hazard Ratio $0.25 \quad 0.50 \quad 1.0 \quad 2.0 \quad 4.0 \quad 8.0$
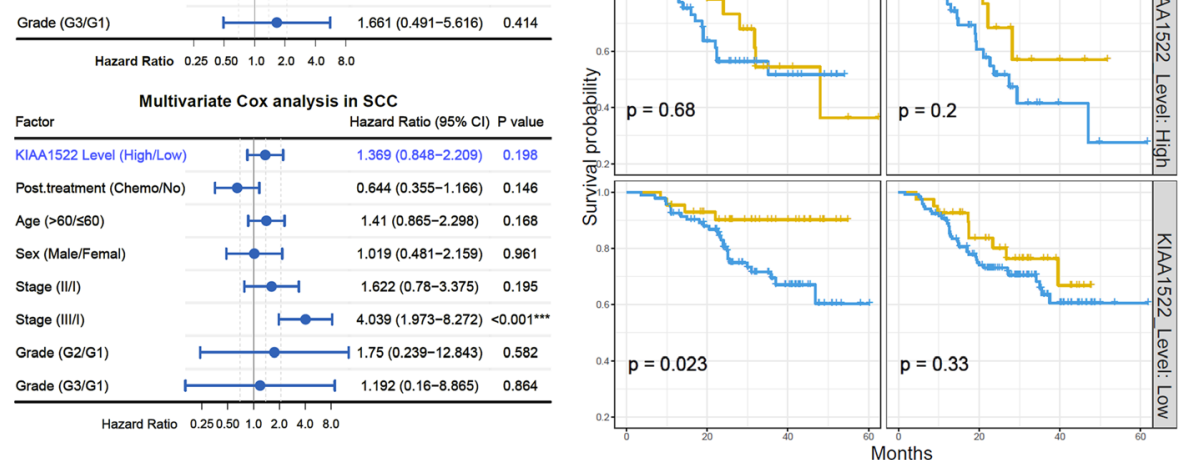

Fig. 1 KIAA1522 expression predicts platinum-based therapeutic effectiveness in lung adenocarcinomas. a Forest plot shows the hazard ratio with 95\% confidence interval of each tested protein. The hazard ratios are estimated by univariate cox analyses in all NSCLC patients (blue) or the patients receiving post-surgery platinum-based chemotherapy (red). The proteins were ranked by the $p$-values in the chemotherapy group. b-f Immunohistochemical analyses of KIAA1522 expression in tissue samples from NSCLC patients. b Representative images of KIAA1522 immunohistochemical staining in NSCLC specimens and non-tumoral normal lung tissues. Bar $=100 \mu \mathrm{m}$. c Paired comparisons of KIAA1522 expression in non-tumoral and tumor samples in either lung adenocarcinomas (ADC) or squamous cell carcinomas (SCC), paired t-test, ${ }^{* * * * P}<$ 0.0001. d In ADC and SCC patients, multivariate cox analyses of prognostic values of KIAA1522 levels excluding age, sex, stage, grade and postsurgical chemotherapy. e Kaplan-Meier curves show the overall survival of NSCLC patients expressing high/low levels of KIAA1522 protein with or without platinum-based post-surgical adjuvant chemotherapy. $\mathbf{f}$ Comparisons of prognostic effects of platinum-based chemotherapy in KIAA1522 high expression or low expression groups respectively

of NSCLC patients, there is no difference in survival rate between histological types in both chemo-naive and chemotherapy-experienced patients (Supplementary Fig. 1E). The platinum-based chemotherapy seems to improve the survival probability, but hardly separate the Kaplan-Meier curves significantly (Supplementary
Fig. 1F). Remarkably, in both ADC and SCC, incorporating the expression levels of KIAA1522 successfully stratified one group of lung adenocarcinoma patients with the most favorable outcomes, who express lower level of KIAA1522 and underwent platinum-based chemotherapy (Fig. 1e). When the NSCLC patients were 
grouped by histological types and KIAA1522 levels, chemotherapy in KIAA1522-low expression group of adenocarcinomas was more efficient than other groups (Fig. 1f). In TCGA lung cancer datasets, we found that the expression of KIAA1522 elevated in both adenocarcinomas and squamous cell carcinomas (Supplementary Fig. 2A), but only in the adenocarcinoma datasets, KIAA1522 expression connected with poor prognosis (Supplementary Fig. 2B). Above all, the clinical analyses highlight the predictive value of KIAA1522 in both overall survival and survival after platinum-based chemotherapy. Meanwhile, the results suggest the tumorpromoting and chemo-resisting roles of KIAA1522 in lung adenocarcinomas.

\section{Depletion of KIAA1522 impairs tumorigenesis and potentiates chemosensitivity in $\mathrm{Kras}^{G 12 D}$-induced murine lung adenocarcinomas}

We employed an oncogenic Kras-induced lung adenocarcinoma model to study whether and to what extend KIAA1522 influence tumorigenesis and chemoresistance. The kiaa1522 specific sgRNAs were integrated together with a Cre expressing cascade into recombinant adeno-associated virus (AAV). Then, the rAAVs were delivered intratracheally to the $L S L$ Cas $9 / L S L$-Kras ${ }^{G 12 D}$ mice (Fig. 2a). The mice delivered by control sgRNA and kiaa1522 sgRNA were divided into two groups treated by vehicle and cisplatin respectively (Fig. 2a). After the mice were sacrificed, the lungs were weighted (Fig. 2b), then subjected to Bouin's staining or HE staining (Fig. 2c). The tumoral lungs in the kiaa1522 sgRNA-introduced mice had reduced lung weights compared to control mice. Upon cisplatin management, the lung weights were further lost in the kiaa1522-edited mice (Fig. 2b). When comparing the tumor burden between groups, we found that down-regulation of KIAA1522 dramatically attenuated $\mathrm{Kras}^{G 12 D}$-induced lung adenocarcinoma in situ. More importantly, the depleting of KIAA1522 synergistically improved the efficiency of cisplatin in shrinking lung tumors (Fig. 2c-e, Supplementary Fig. $2 \mathrm{C})$. The following survival analysis also substantiated the benefits of KIAA1522 down-regulation which not only elevated the survival rate of tumorigenic mice but also made them more sensitive to cisplatin therapy (Fig. 2f). Coincidently, the combination of KIAA1522 depletion and cisplatin treatment considerably induced apoptosis in vivo, monitored by immunohistochemical staining of cleaved-caspase 3 (Fig. 2g).

We also down-regulated KIAA1522 expression in the established lung adenocarcinoma cell lines and analyzed by their tumorigenic capability and cisplatin response. The results showed that loss of KIAA1522 severely impaired subcutaneous tumors (Fig. 3a-b), and reduced the amount of Ki67 positive cells in vivo (Fig. 3c). Moreover, down-regulation of KIAA1522 reduced the IC50 value in response to cisplatin (Fig. 3d). The KIAA1522 depleted cells were more sensitive to cisplatin treatment in vitro (Fig. 3e). Oppositely, overexpression of KIAA1522 rendered the cells chemoresistance to cisplatin (Fig. 3f).

The above results strongly indicate that KIAA1522 is required for both the tumorigenesis and resistance to cisplatin in lung adenocarcinoma, these facts coincide with the clinical relevance of KIAA1522 in lung adenocarcinoma patients.

\section{KIAA1522 promotes the TNFa-NFKB signaling pathway}

To understand the molecular basis of KIAA1522mediated acquirement of chemoresistance to cisplatin, we performed transcriptional profiling assays to detect dysregulated genes upon KIAA1522 depletion in 889 lung adenocarcinoma cells (Fig. 4a). Enrichment analysis of KIAA1522 positive regulated genes using HALLMA RK signatures showed that the geneset HALLMARK TNFA_SIGNALING_VIA_ NFKB pathway was on the top of enriched pathways (Fig. 4b), suggesting that KIAA1522 may enhance TNF $\alpha-N F \kappa B$ signaling pathway. This conclusion was further strengthened by gene set enrichment analysis (GSEA) and gene set variation analysis (GSVA) analysis. GSEA and GSVA results both showed that TNF $\alpha-N F K B$ signaling associated genesets were down-regulated in the KIAA1522 depletion cells (Fig. 4c-d). Similar to TNFo-NFkB signatures, The GSVA scores of cisplatin resistance signatures and cisplatin-responsive signatures were also down-regulated in the 889 cells expressing sg-kiaa1522. While the gene signatures negatively related to cisplatin resistance were up-regulated in KIAA1522 down-regulated cells (Fig. $4 \mathrm{e})$.

In the TCGA-LUAD dataset, when considering KIAA1522 expression (KIAA1522 mRNA) and TNFa/ NFkB status together, the patients with low KIAA1522 and TNF $\alpha$ signal levels or low KIAA1522 and NFkB activity had the best outcomes (Supplementary Fig. 3A; Fig. 4f). In addition, we also observed the correlation of KIAA1522 expression with both TNF signaling (Supplementary Fig. 3B), and NFkB activity (Fig. 4g) in the GSEA assays. TNF $\alpha-N F \kappa B$ signaling has been reported to cause cisplatin insensitivity in several types of malignancies [13]. We found that inhibition of KIAA1522 reduced a set of genes down-stream of $\mathrm{NFK}_{\mathrm{K}}$ signaling, including anti-apoptotic genes confering cisplatin resistance, such as Xiap, Bcl2 and Birc5/survivin [15] (Supplementary Fig. 3C). So, the hyperactivation of TNF $\alpha-\mathrm{NF}_{\kappa} B$ signaling may be a key molecular event responsible for KIAA1522-induced counteractivity to cisplatin. 

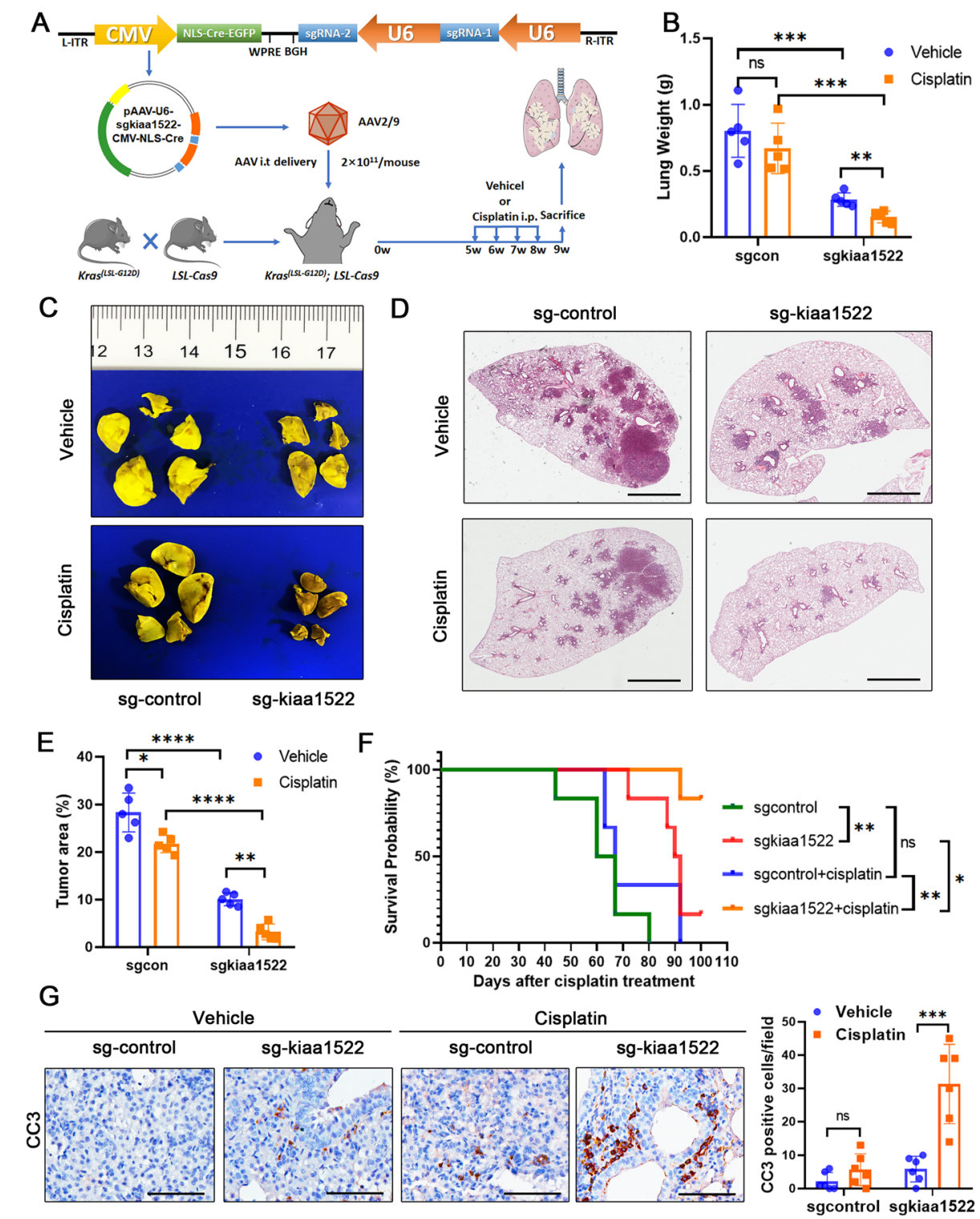

Fig. 2 Genetically depletion of kiaa1522 gene impairs Kras ${ }^{G 12 D}$-induced lung adenocarcinoma and sensitizes tumors to cisplatin. a Schematic showing of adenovirus associated virus (AAV)-mediated kiaa1522 depletion in $\mathrm{Kras}^{\text {G12D-induced lung. The Kras }}{ }^{\text {(LLL-G12D), }}$ LSL-Cas9 mice were intratracheal administrated by Cre- and control sgRNA or kiaa1522 sgRNAs-expressing-AAVs. Then, the mice were subjected to i.p. injections of vehicle/cisplatin. $\mathbf{b}$ The comparison of lung weights in different groups. t-test, ${ }^{* *} P<0.01,{ }^{* * *} P<0.001$. c-d Representative image of the lung after Bouin's staining (c) and HE staining (d) in each group. e The comparison of tumor area in different groups. t-test, ${ }^{*} P<0.05$, ${ }^{* * *} P<0.0001$. Bar $=2$ mm. $\mathbf{f}$ Kaplan-Meier curves show the role of KIAA1522-depletion and/or effect of cisplatin on the outcome of lung cancer in mice. The survival rates were compared between each group. Log-rank test, ${ }^{*} P<0.05$, ${ }^{*} P<0.01$. g Immunohistochemical staining of cleaved-caspase 3 in the lung tumor samples from the indicated mice. t-test, ${ }^{* * *} p<0.001 . \mathrm{Bar}=100 \mu \mathrm{m}$

We next explore whether KIAA1522 promotes $\mathrm{TNF} \alpha-\mathrm{NF} K \mathrm{~B}$ signaling transduction in the cultured cells. We found that down-regulation of KIAA1522 by shRNA or Cas9/sgRNA substantially inhibited the basal levels of phosphorylated NF-kB p65/RelA (Fig. 5a, Supplementary Fig. 4A). In the presence of exogenous TNFa to stimulate NFKB signaling in the serum-deprived cells, loss of KIAA1522 remarkably alleviated $\mathrm{NF}_{K} \mathrm{~B}$ signaling activity monitored by luciferase reporter (Supplementary Fig. 4B). Western blotting assays showed that TNF $\alpha$ was less effective to up-regulated the phosphorylation of $\mathrm{p} 65, \mathrm{IKK} \alpha / \beta$ and ІкB in the KIAA1522-downregulated cells (Fig. 5b-c, Supplementary Fig. 4C-D), whereas forced expression of KIAA1522 yielded an opposite effect, which expedited the activation of $\mathrm{NFKB}$ signaling (Fig. 5d, Supplementary Fig. 4E). Moreover, we found that depletion of KIAA1522 decreased the 

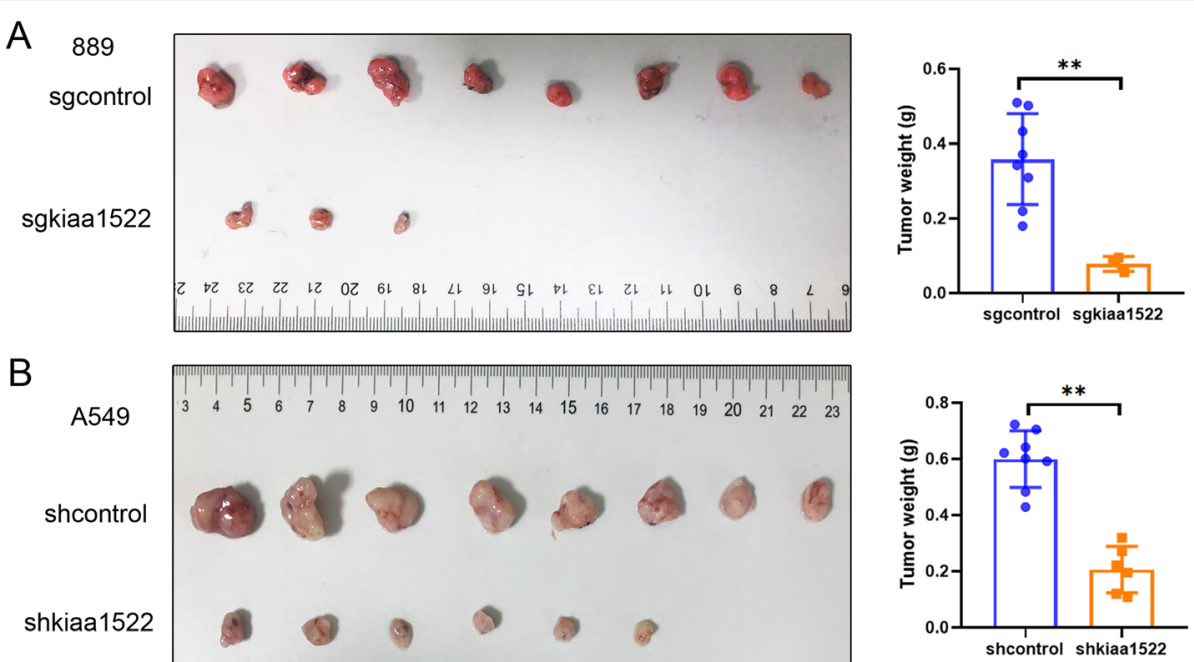

C

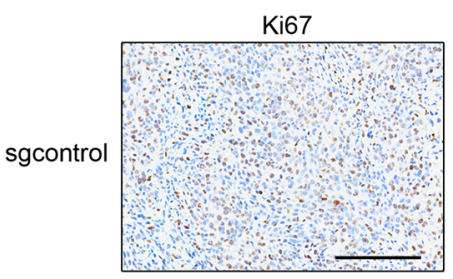

D 889
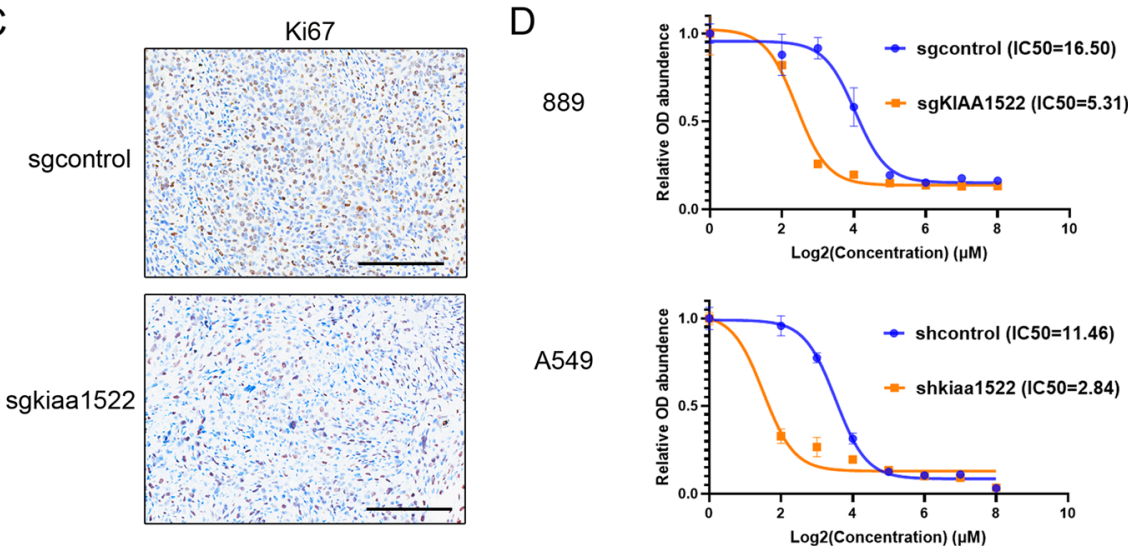

$E$ A549
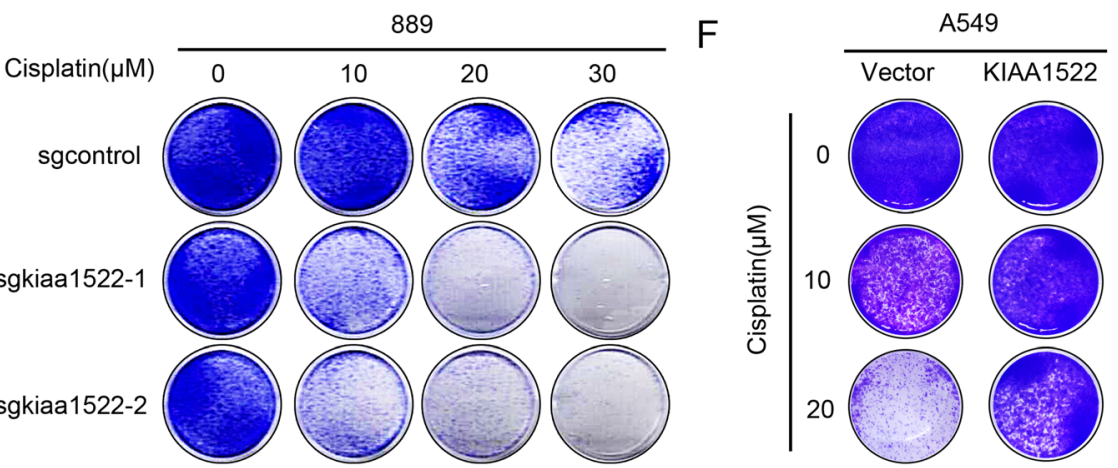

Fig. 3 Depletion of KIAA1522 in lung adenocarcinoma cells reduces tumor growth and resistance to cisplatin. a Murine cell line 889 with the sgRNA-mediated depletion of KIAA1522 were injected into C57BL/6 J mice subcutaneously. One month later, the mice were sacrificed and the tumor weights were quantified. t-test, ${ }^{* *} P<0.01$. b Human adenocarcinoma cell A549 expressing KIAA1522-shRNA were injected into nude mice subcutaneously. After two months, the mice were subjected to tumor weights quantification. t-test, $* * P<0.01$. c Immunohistochemical staining of Ki67 in control sgRNA/Cas9 or kiaa1522-sgRNA/Cas9 expressing 889 cells. Bar $=100 \mu \mathrm{m}$. d IC50 of cisplatin in both 889 cells and A549 cells with down-regulated KIAA1522 were analyzed by CCK8 assays. e-f The inhibitory effects of cisplatin on 889 cells expressing kiaa1522-sgRNA/Cas9 (e) or A549 cells overexpressing KIAA1522 (f) by colony formation assays

abundance of the Tumor Necrosis Factor receptors, TNFR1 and TNFR2 (Fig. 5e, Supplementary Fig. 4FG). Overexpression of KIAA1522 significantly increased TNFR2 in parallel with the activation of p65, but not TNFR1 (Fig. 5f, Supplementary Fig. 4H). So, the up-regulation of TNFR2 may contribute to the KIAA1522-mediated TNF $\alpha-N F K B$ activation. The AAV-mediated deletion of KIAA1522 in $\mathrm{Kras}^{\mathrm{G12D}}$-induced murine lung cancer also repressed TNFR2 and $\mathrm{NF}_{\mathrm{K} B}$ signaling, as indicated by phosphorylated 
A

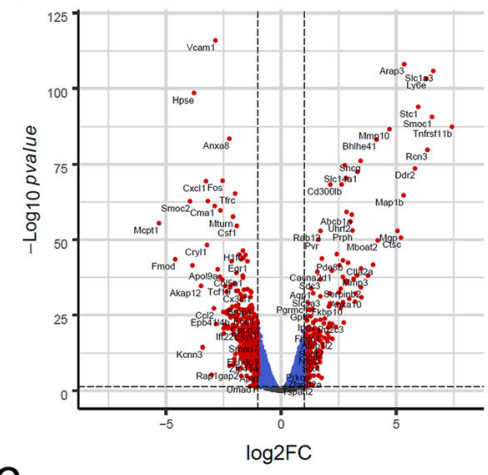

C

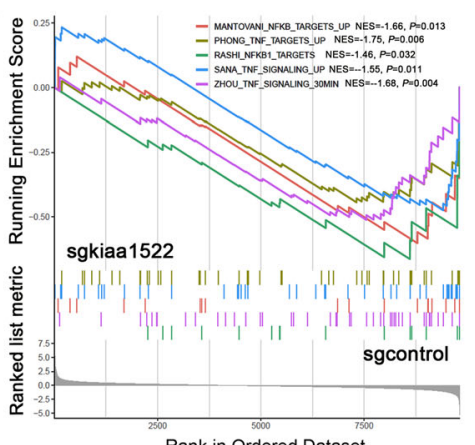

E

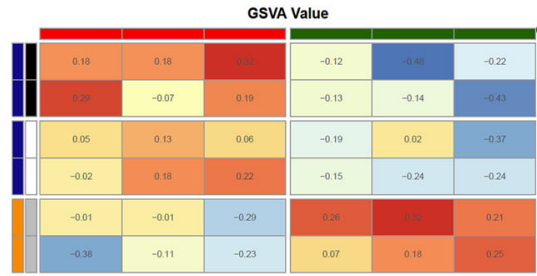

B

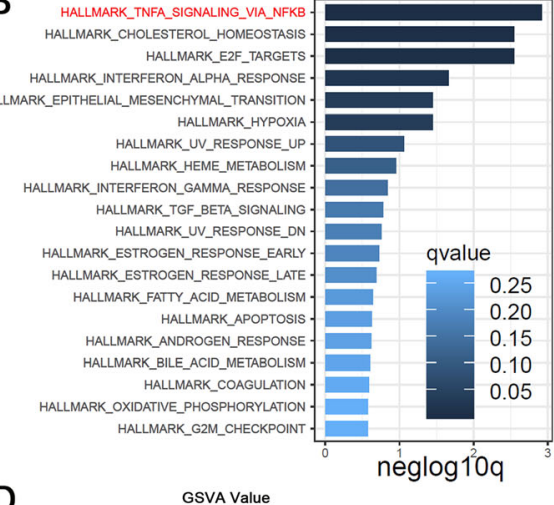

D
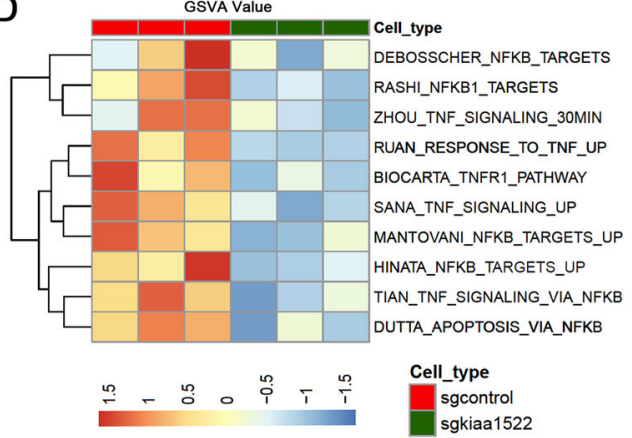

Cell_type

LI_CISPLATIN_RESISTANCE_UP

TSUNODA_CISPLATIN_RESISTANCE_UP

BRACHAT_RESPONSE_TO_CISPLATIN

KERLEY_RESPONSE_TO_CISPLATIN_UP

LI_CISPLATIN_RESISTANCE_DN

KANG_CISPLATIN_RESISTANCE_DN
Cell_type

0.2 sgcontrol

sgkiaa 1522

sgkiaa1522_vs_con

DOWN

UP

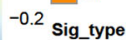

pos_cisplatin_resistance

-0.4 cisplatin_response neg_cisplatin_resistance
$\mathbf{F}$

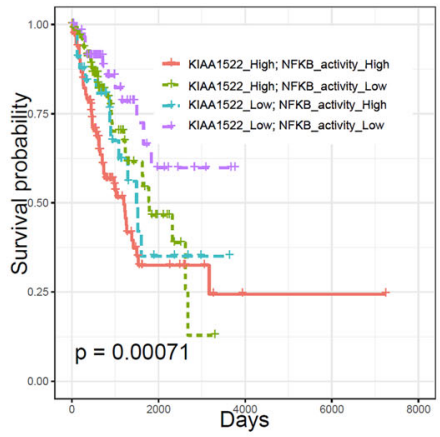

G

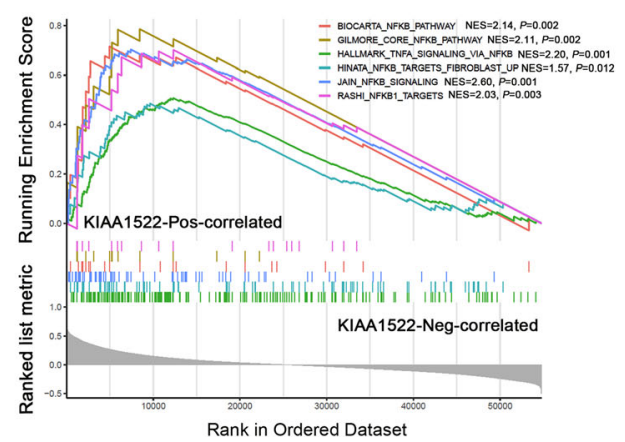

Fig. 4 KIAA1522 regulates gene expression signatures associated with TNF-NFKB pathway and cisplatin resistance. a-e RNA-sequencing experiments were performed to detect the expression profiling of control and 889 cells depleting of KIAA1522. Each group includes three replicates. a volcano plot shows the different expressed genes of KIAA1522 depleted 889 cells compared to the control cells. The genes with both $\left|\log _{2} \mathrm{FC}\right|>2$ and $p$-value $<0.05$ were considered as significantly differentiated genes. $\mathbf{b}$ Enrichment analysis of KIAA1522 positively regulated genes in HALLMARK gene signatures, the significantly enriched signatures were ranked by q-values, the bar plot shows the top 20 signatures. $\mathbf{c}$ Gene sets enrichment analysis (GSEA) of genes by ranked by $\log _{2}$ FC (sgkiaa1522/ sgcontrol) values using a set of TNF-NFKB pathway related signatures. $\mathbf{d}$-e The GSVA score of the genesets related to the TNF-NFKB pathway (d) and experiments derived cisplatin-resistant gene signatures (e) were determined by GSVA algorithm. The heatmaps show the distribution of GSVA values between the indicated group of cells. $\mathbf{f}$ The patients in TCGA-LUAD cohort were classified by both KIAA1522 expression and NFKB activity into four groups and subjected to Kaplan-Meier overall survival analysis. The activity of NFKB signaling was determined by the GSVA score of the geneset JAIN_NFKB_SIGNALING. g The genes were ranked by the Pearson correlation coefficient with KIAA1522 expression in TCGA-LUAD datasets. Then, the GSEA assays were performed using a set of NFKB activity positively associated signatures to evaluate the correlation of KIAA1522 expression level with NFKB signaling activity 


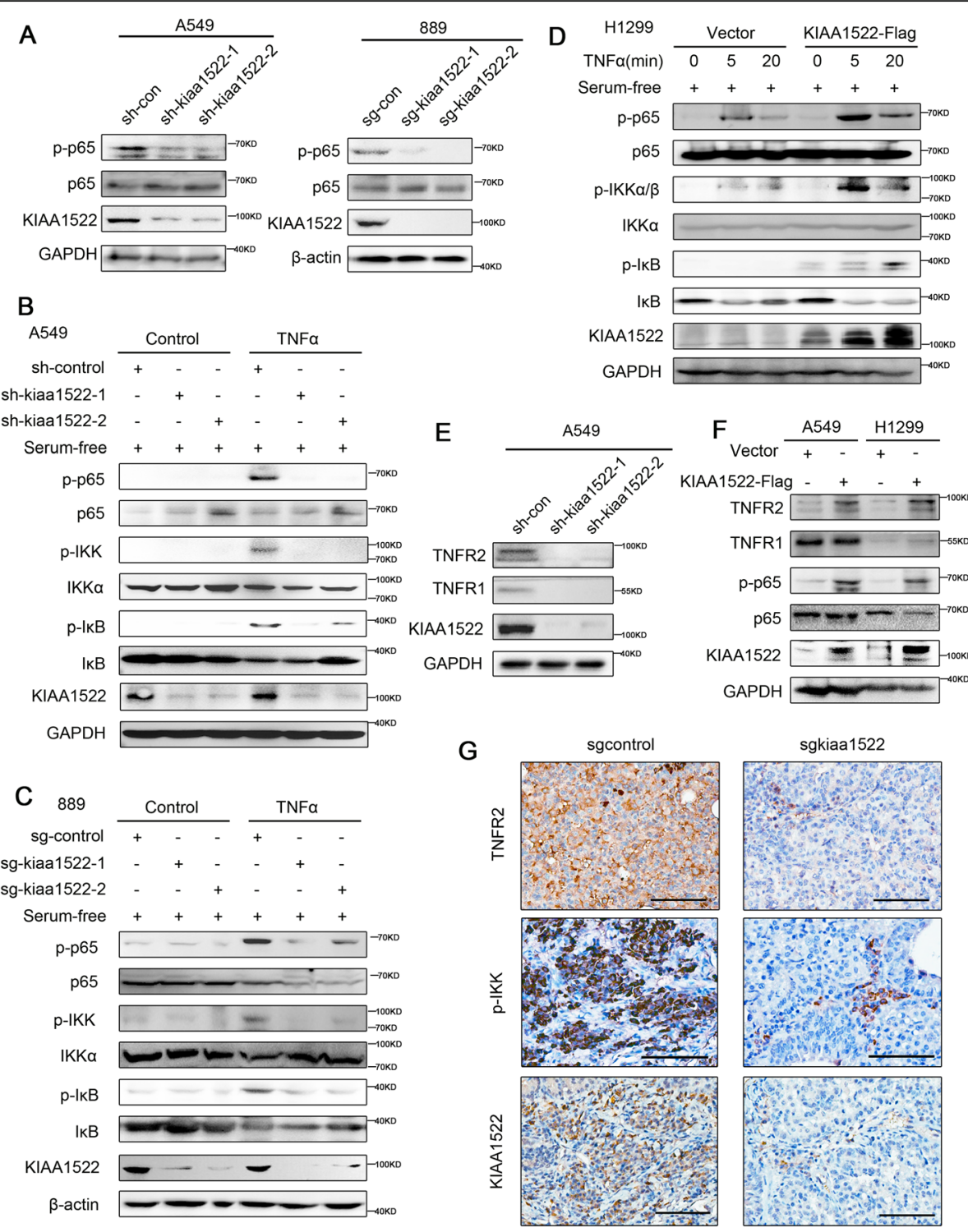

Fig. 5 KIAA1522 positively regulates TNFa-NFKB signaling in lung adenocarcinoma cells. a Immunoblotting assays of Phospho-NFkB p65 (Ser536) in the control and KIAA1522 down-regulated cells. b-c The indicated group of A549 cells (b) or 889 cells (c) were deprived of serum for $20 \mathrm{~h}$, then treated with TNFa for $5 \mathrm{~min}$. Then the cells were tested by Western blotting assay to detect the phosphorylation of p65, IKK and IKB proteins. d H1299 cells overexpressing Flag tagged KIAA1522 protein were cultured in serum-free condition for $20 \mathrm{~h}$, then treated by TNFa and harvest at the indicated time point. The harvested cells were subjected to Western blotting assay. e Western blotting assays show the expression of TNFR2 and TNFR1 in KIAA1522 down-regulated A549 cells. f Western blotting assays show the expression of TNFR2 and TNFR1 in KIAA1522overexpressed cells. $\mathbf{g}$ Immunohistochemical staining of TNFR2, phosphorylated IKKa/ $\beta$ and KIAA1522 in Kras-induced murine lung tumor samples with or without KIAA1522 depletion. Bar $=100 \mu \mathrm{m}$

$\mathrm{IKK} \alpha / \beta$ (Fig. $5 \mathrm{~g}$ ). The results verified the regulation of TNF $\alpha-N F K B$ pathway in vivo. Next, we also explore the potential mechanisms underlying KIAA1522 regulating TNFR2, we found that depletion of KIAA1522 did not decrease the mRNA expression of KIAA1522 (Supplementary Fig. 5A), while KIAA1522 interacted with TNFR2 (Supplementary Fig. $5 \mathrm{~B}$ ) and contributed to protein stability of TNFR2 (Supplementary Fig. 5C).
Correlation of KIAA1522 signature with TNFa-NFKB signaling and cisplatin responsiveness in multiple cohorts of lung adenocarcinoma patients

To further underpin the links between KIAA1522 and TNF $\alpha-N_{F} B$ signaling and cisplatin responsiveness by clinical evidences, we performed integrative analyses using transcriptome profiles of lung adenocarcinoma patients from multiple cohorts, including single cell transcriptome studies. Considering the lake of KIAA1522 
probes in several platforms, we defined a KIAA1522 POS_REG_GENES geneset by KIAA1522 positive regulated genes in 889 cells (Fig. 4a) to calculate KIAA1522 signature score representing KIAA1522 expression amount, in bulk- or single cell-level transcriptome assays.

In the lung cancer single cell transcriptome dataset EMTAB-6149, we clustered the cells by Seurat software (Supplementary Fig. 6A) and filtered tumor cells by the expression of EpCAM (Supplementary Fig. 6B). Then the clusters were further classified by the expression of ADC marker NAPSA/ Napsin A and SCC marker TP63 / P63. Some groups of cells expressing NAPSA and negative for TP63 were identified as lung adenocarcinoma cells (Fig. 6a). Before in-depth analyses, we examined the reasonability of using the GSVA scores derived from KIAA1522 downstream signatures. Firstly, in the sorted adenocarcinoma cells, we observed that the KIAA1522 signature scores were much higher in the KIAA1522 mRNA positive cells than that in KIAA1522 negative cells, suggesting the consistency of KIAA1522 mRNA levels and KIAA1522 signature scores (Fig. 6b). Secondly, we analyzed the clinical relevance of cisplatin resistance signatures and found that the two selected signatures were both correlated with poor survival in the lung adenocarcinoma patients after cisplatin-based therapy (Supplementary Fig. 6C). indicating these GSVA scores recapitulated physically resistance to cisplatin. Pearson correlation analyses indicated that the KIAA1522 signature scores were correlated with the TNF $\alpha$ signaling, NFKB activity and cisplatin resistance associated signatures (Supplementary Fig. 6D). The 3D plot clearly exhibited that the single cells with high levels of KIAA1522 signature scores were hyperactivated in $\mathrm{TNF} \alpha-\mathrm{NF} \mathrm{KB}$ signaling and highly resistant to cisplatin simultaneously (Fig. 6c). Besides the representative genesets, we also showed that the KIAA1522 scores were highly consistent with the GSVA scores of a handfuls of TNF $\alpha-N F \kappa B$ associated signatures (Fig. 6d).

In the bulk RNA sequencing dataset TCGA-LUAD, we also observed a similar distribution of the scores of all the signatures tested including the signatures related to TNF $\alpha-N F k B$ signaling, cisplatin resistance and KIAA1522 signature scores (Supplementary Fig. 7A). To strengthen this result, systematic correlation analyses showed that the positive correlations were universal in multi-central datasets (Fig. 6e). The correlations of KIAA1522 signature scores with TNF $\alpha-\mathrm{NF}$ BB- cisplatin resistance signatures were independent of oncogenic KRAS and TP53 mutations (Supplementary Fig. 5B). Furthermore, meta-analysis was performed to review the prognostic effects of KIAA1522 signature score in independent datasets with survival information. The results showed that high level of KIAA1522 signature score predicted poor outcome in most of the datasets (Fig. 6f). The fixed effects model estimated hazard ratio was 1.918 (1.723-2.113) with a $P$-value $<0.001$, this is in agreement with the conclusion detected in our cohorts of patients. More importantly, there are 39 cases in the dataset GSE14814 received platinum-based adjuvant chemotherapy. In this specific cohort, lower level of KIAA1522 scores also implicated good therapeutic results (Fig. 6g). When considering the combined effects of KIAA1522 score and NFkB status, the result showed that the groups of patients with both low levels of KIAA1522 and NFKB activity had the best outcomes (Fig. 6h-i).

\section{Inhibition of NFKB signaling restores sensitivity to cisplatin in KIAA1522 overexpressed cells}

To test whether hyperactivation of NFkB signaling is critical for the KIAA1522-induced chemoresistance, we used a NFKB inhibitor QNZ to block NFאB signaling activity (Fig. 7a). QNZ ameliorated the irresponsiveness of the exogenous KIAA1522 expressed cells (Fig. 7b-c). Coherently, in the presence of QNZ, either the control cells or the KIAA1522-depleted cells yielded similar effects in response to cisplatin (Fig. $7 \mathrm{~d}$-e). These results substantiated that NFKB signaling is engaged in KIAA1522 mediated cisplatin resistance, and enlightened us to cooperatively use of $\mathrm{NF}_{\kappa} \mathrm{B}$ inhibitor and cisplatin to circumvent the treatment-refractory nature of lung adenocarcinomas expressed high level KIAA1522. To exam this speculation, we performed the in vivo pharmacology experiment. The results revealed that although forced expression of KIAA1522 did not accelerate tumorigenesis, but it desensitized inhibitory effects of cisplatin in vivo. The KIAA1522-induced cisplatin resistance, like the in vitro observations, dampened by NFKB inhibition (Fig. 7f). Collectively, synergic usage of NFKB inhibitor and cisplatin counteracted the cisplatin-refractory phenotype of KIAA1522 overexpressed lung adenocarcinoma cells.

\section{Discussion}

Nowadays, platinum-based adjuvant chemotherapy is still an irreplaceable way in routine practice to treat NSCLC patients after curative resection. Nevertheless, the efficiency is quite limited. One ideal route to optimize the usage of platinum reagents relies on customizing the patients based on histological diagnosis and the expression of tumor biomarkers [35]. Histological subtypes of non-small cell lung cancer (ADC or SCC) determine the cisplatin responsiveness [12], choice of treatments [36], or the dependency of biomarkers [37]. But effective indicators for each type of NSCLC are urgently needed. In our cohort of NSCLC patients, it is similar in the survival rate of ADC and SCC in either chemo-naive or chemotherapy-receiving 

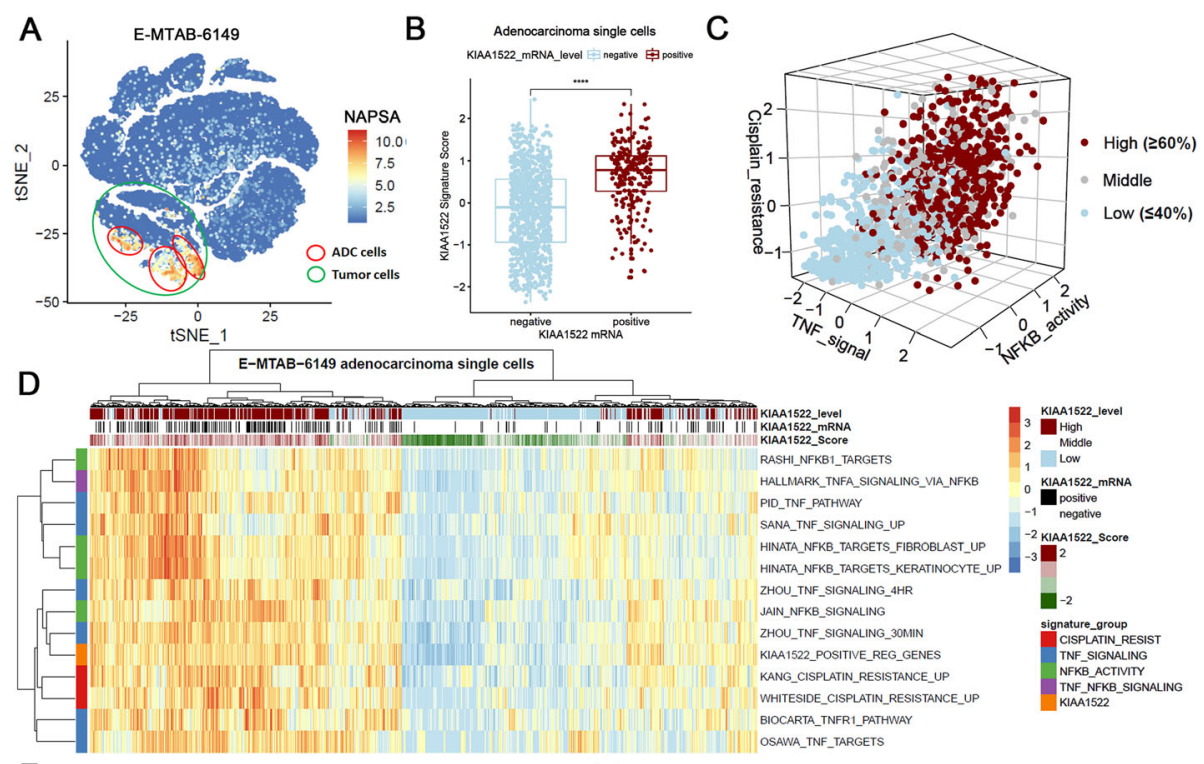
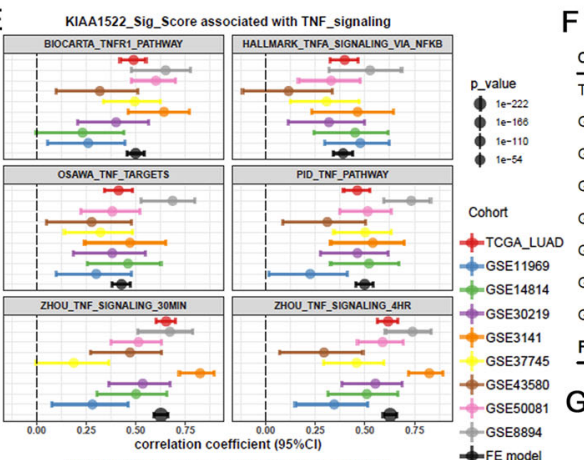

PFE mode

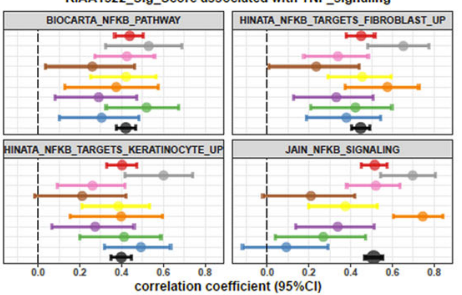

KIAA1522_Sig_Score associated with TNF_signalin

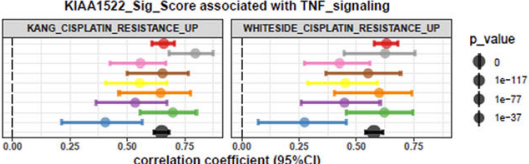

F Prognositc effects of KIAA1522_Signature_Score: Meta-analysis

\begin{tabular}{|c|c|c|c|c|c|}
\hline Cohort & Type/Treat & & Cases (\%) & HR $(95 \% \mathrm{Cl})$ & $P$ value \\
\hline$\overline{\text { TCGA_LUAD }}$ & DADC/NO & $1-1$ & $533(43.263)$ & $1.568(1.151-2.135)$ & 0.004 \\
\hline GSE31210 & ADC/NO & $\longmapsto$ & $-226(18.344)$ & $3.577(1.459-8.770)$ & 0.005 \\
\hline GSE13213 & ADC/NO & $\longmapsto$ & $117(9.497)$ & $1.930(1.071-3.477)$ & 0.029 \\
\hline GSE 37745 & ADC/NO & $\mapsto 1$ & $106(8.604)$ & $1.654(1.016-2.691)$ & 0.043 \\
\hline GSE11969 & ADC/NOH & $\rightarrow-1$ & $90(7.305)$ & $1.545(0.820-2.913)$ & 0.179 \\
\hline GSE8894 & ADC/NO & $\longmapsto 1$ & $63(5.114)$ & $2.270(1.051-4.904)$ & 0.037 \\
\hline GSE3141 & $\mathrm{ADC} / \mathrm{NO}$ & $\longmapsto$ & $58(4.708)$ & $3.143(1.206-8.196)$ & 0.019 \\
\hline GSE14814 & $A D C / A C T$ & $\longmapsto$ & $39(3.166)$ & $3.503(1.438-8.534)$ & 0.006 \\
\hline FE model & & - & $1232(100) 1$. & $1.918(1.723-2.113)$ & $<0.001$ \\
\hline
\end{tabular}

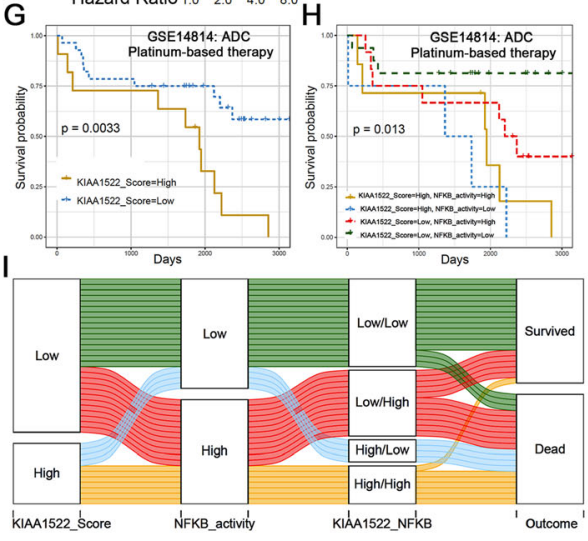

Fig. 6 KIAA1522 signature correlates with TNF-NFKB-Cisplatin resistance in independent cohorts of adenocarcinomas. The KIAA1522 signature was generated by the collection of KIAA1522 down-stream genes and transformed to KIAA1522 signature score through GSVA algorithm. a Single-cell sequencing data from E-MTAB-6149 dataset was subjected to tSNE dimension reduction and clustering the NAPSA positive clusters were shown. $\mathbf{b}$ The KIAA1522 signature scores in KIAA1522 mRNA positive and negative adenocarcinoma single cells were compared, t-test, ${ }^{* * * * P}<0.0001$. c 3D plot shows the distributions of KIAA1522 score levels according to TNFa signaling score, NFKB activity score and cisplatin resistance score. $\mathbf{d}$ Heatmap shows the scaled GSVA score of a collection of TNF-NFKB-Cisplatin resistance signatures in adenocarcinoma single-cells within E-MTAB-6149 dataset in parallel with KIAA1522 mRNA expression and KIAA1522 signature score. e In multi-central ADC samples, meta-analytical evaluation of the correlation between KIAA1522 signature score with TNF signaling, NFkB activity and cisplatin resistance signatures. Forest plots show the correlations together with the $95 \%$ confidence intervals, and the fixed-model estimated correlation in each geneset. $\mathbf{f}$ Systematical univariate cox analyses estimate the Hazard Ratio of KIAA1522 signature score in multiple datasets from TCGA or GEO databases. The meta-analysis was performed by fixed model to estimate the prognostic effect of KIAA1522 signature score. $\mathbf{g}$-h In GSE14814 datasets, adenocarcinoma patients receiving platinum-based chemotherapy were grouped according to KIAA1522 signature score alone $(\mathbf{g})$ or the combination of KIAA1522 signature score and NFKB activity score (h), then survival rates of each group were revealed by Kaplan-Meier survival analyses. i Alluvial diagram of KIAA1522 signature score, NFKB activity, combined KIAA1522_NFKB score and the survival outcome 
A

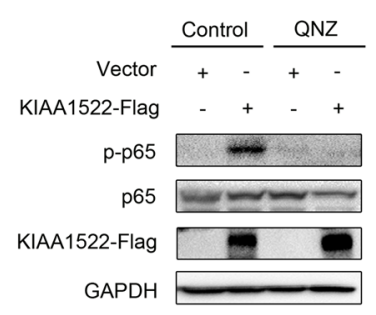

B

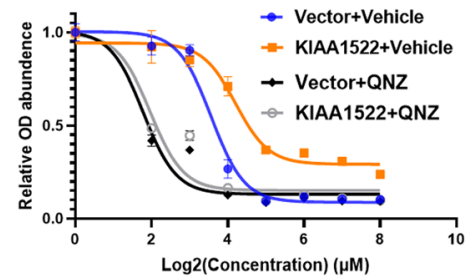

$\mathrm{C}_{\mathrm{A} 549}$

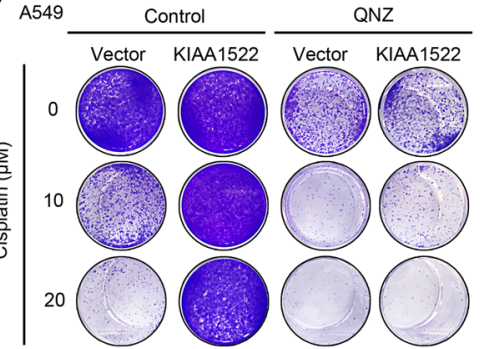

H129

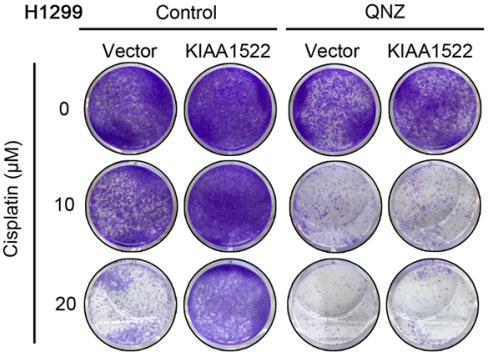

D

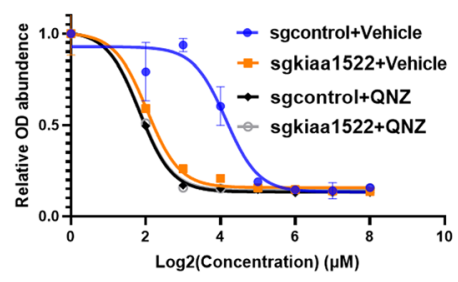

E
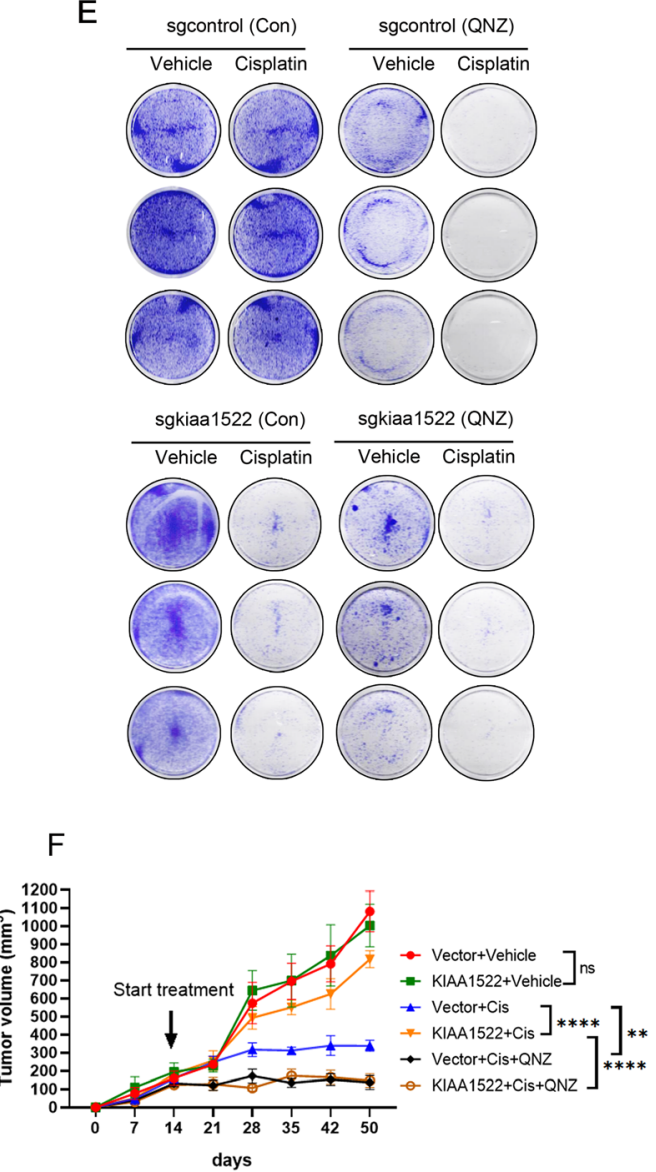

Fig. 7 Inhibition of NFKB signaling counteracts cisplatin insensitivity in KIAA1522 hyperactivated cells. a Inhibitory effect of QNZ on NFKB signaling in KIAA1522-overexpressed A549 cells was tested by immunoblotting assay. b The control and KIAA1522-overexpressed A549 cells were treated by indicated concentration of cisplatin with or without QNZ (500 nM). Cell viability were analyzed by CCK8 assays. c Clonal formation assays measuring the effect of QNZ on cisplatin-induced growth inhibition in the control and KIAA1522-overexpressed A549 and H1299 cells. The cells were treated with $500 \mathrm{nM}$ QNZ and the indicated concentration of cisplatin for $48 \mathrm{~h}$ before crystal violet staining. $\mathbf{d}$ The control and KIAA1522 depleted 889 cells were treated by different concentrations of cisplatin with or without QNZ, then analyzed by CCK8 assays to determine cell viability. e Clonal formation assays of the control-sgRNA/Cas9 and kiaa1522-sgRNA/Cas9 expressing 889 cells treated by QNZ and cisplatin for $48 \mathrm{~h}$. $\mathbf{f}$ the control and KIAA1522-overexpressed A549 cells were subcutaneously injected into nude mice. From 14 days after cell injection to the 50th day, the mice were treated by cisplatin (7 mg/kg B.W.) once a week and QNZ (1 mg/kg B.W.) twice a week via intraperitoneal injection. Tumor volumes in the last day were compared using t-test, ${ }^{* *} P<0.01,{ }^{* * * *} P<0.0001$

patients (Supplementary Fig. 1E). Although the treatment by platinum-based chemotherapy slightly alleviated tumoral death, but failed to reach the statistical threshold (Supplementary Fig. 1F). In this background, it is meaningful to find that the expression of KIAA1522 characterizes a hypersensitive group in the lung adenocarcinoma, emphasizing KIAA1522 a potential indicator to forecast therapeutic consequence of platinum-based chemotherapy. Moreover, we found that in the GSE14814 dataset [38], containing expression profiles of ADC patients receiving cisplatin- doublet chemotherapy, KIAA1522 acts in a similar degree to predict therapeutic efficiency, supporting the robustness of this predictor. 
Previously, we have reported that the expression of KIAA1522 was altered even in early lung tumor cells in bronchial brushing specimens [21]. It can be readily detected using commercial antibodies. The clear immunohistochemical staining signal enables KIAA1522 suitable for pathological diagnosis in the clinical application, that is the prerequisite in selecting candidate proteins for our clinical analyses. In contrast to the strong staining signal in tumoral tissues, the expression of KIAA1522 were extremely low in nearly 500 non-tumoral lung tissues in our cohort, as well as in the TCGA datasets. We have also identified KIAA1522 as a prognostic factor for NSCLC in a former study [22]. Here we elucidate the predictive role of KIAA1522 detailly with the verification in multicentered independent cohorts. Unlike the controversial results from squamous cell carcinoma, the prognostic values of KIAA1522 in lung adenocarcinoma were highly consistent in almost all studied datasets from several databases. The convincing clinical results implied the pleiotropic roles of this gene participating in variant steps of lung adenocarcinoma, and encourage us conducted the functional studies about KIAA1522. To do this robustly, we employed a $\mathrm{Kras}^{G 12 D}$-induced murine lung adenocarcinoma model coupled by Cas9/sgRNA-mediated gene editing [39]. This in vivo system easily generated lung cancer cell specific-knockout mice, empowering the genetical evaluation of KIAA1522 function in an economic effective manner. The in vivo experiments using genetic mice model represent the clinical results, reinforcing the requirement of KIAA1522 to both tumor development and intrinsic resistance to cisplatin.

Despite the extraordinary biological effects of KIAA1522 in functional assays in vitro and in vivo, the molecular details of this protein remain elusive. KIAA1522 is neither an enzyme or a receptor, making it undruggable based on the existing knowledge. However, uncovering a targetable molecular event key to KIAA1522-downstream pathways may open an alternative avenue to fight against KIAA1522regulated malignancy. To this end, we identified the activation of NFKB signaling was engaged in KIAA1522mediated cisplatin-resistance. Notoriously recognized the significance of $\mathrm{NFK}_{\mathrm{K}} \mathrm{B}$ pathway in distinct biological processes [13], so it needs to be tightly controlled to ensure appropriate onset. As a bona fide upstream activator to stimulate $\mathrm{NF}_{\mathrm{K}} \mathrm{B}$ signaling, TNF $\alpha$ binds to the TNFR1 or TNFR2 receptor complexes to invigorate $N F K B$ signal transduction [40]. Here, we found that KIAA1522 may modulating NFKB activity via TNFR2, a TNF $\alpha$ receptor transmitting only anti-apoptotic signals [41], that is in concordance with the roles of KIAA1522 in lung adenocarcinomas. Further results suggested that the KIAA1522-mediated up-regulation of TNFR2 may occur in the post-transcriptional level, that is KIAA1522 interacting and stabilizing TNFR2. Since our knowledge about the molecular function of KIAA1522 protein were limited, and little is known about the turnover mechanism of TNFR2 protein. So, whether and how KIAA1522 directly work on TNFR2 to active NFkB signal was still far from clear. This is a major limitation of this work that need further exploration. Nonetheless, our pre-clinical studies prove that it is reasonable to inhibit $\mathrm{NFKB}$ activity to reverse the recalcitrant effects of KIAA1522 overexpressed cancer cells. On the other hand, the findings shed light on novel NFKB regulatory mechanism and clinical implication.

Collectively, this work proposes a rational strategy to characterize and treat lung adenocarcinomas. The protein levels of KIAA1522 should be firstly detected as a biomarker to stratify the patients into cisplatin sensitive and insensitive groups. The patients expressed low level of KIAA1522 may be prone to benefit from conventional platinum-based adjuvant chemotherapy. In the opposite, the lung adenocarcinomas with high KIAA1522 expression may be more willing to escape from cisplatin induced regression, which should be treated synergistically by $\mathrm{NF}_{\mathrm{K}} \mathrm{B}$ inhibitor and platinum-based reagents to restore chemosensitivity. This methodology may help to magnify the therapeutic efficiency of platinum-based chemotherapy.

\section{Conclusions}

We found that KIAA1522 acts as an indicator of poor outcome of platinum-based therapy in lung adenocarcinomas. KIAA1522 potentiates the TNF $\alpha-N F k B$ signaling which leads to cisplatin resistance. Our findings suggest that combined use of $\mathrm{NFKB}$ inhibitor and platinumcontained compounds may be active against KIAA1522 overexpressed lung adenocarcinomas.

\section{Supplementary information}

Supplementary information accompanies this paper at https://doi.org/10. 1186/s13046-020-01684-x.

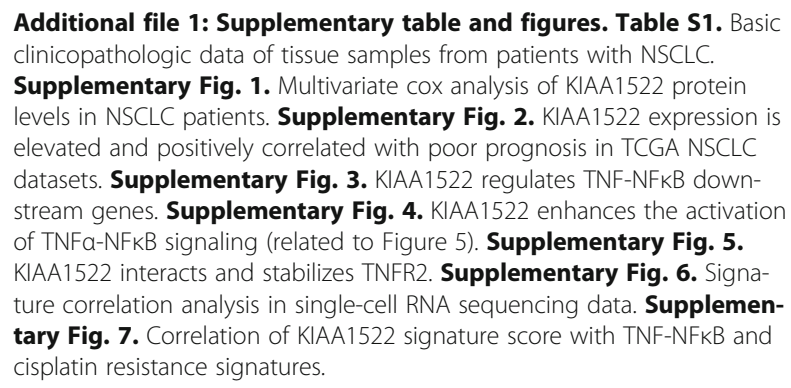

Abbreviations

NSCLC: Non-small cell lung cancer; ADC: Lung adenocarcinomas; SCC: Squamous cell carcinomas (SCC); GSEA: Gene set enrichment analysis; GSVA: Gene set variation analysis analysis; AAV: Adeno-associated virus 


\section{Authors' contributions}

WBS and LYZ designed the research, analyzed data and wrote the manuscript; WBS, JTT, CJN, WCS and LYZ performed the experiments; JWL and WMR contributed reagents and provided intellectual inputs. The author(s) read and approved the final manuscript.

\section{Funding}

This work was supported by the National Natural Science Foundation of China (81702259, 81972209, 81672359), Shanghai Municipal Commission of Health and Family Planning Fund (20174Y0075), and Fudan University Shanghai Cancer Center Fund (YJ201508).

\section{Availability of data and materials}

The datasets generated and/or analyzed during the current study are available in the Gene Expression Omnibus repository (GEO; https://www.ncbi. nlm.nih.gov/geo/) through GEO accessions: GSE146072, GSE3141, GSE8894, GSE13213, GSE11969, GSE37745, GSE31210, GSE30219, GSE50081, GSE43580 and GSE14814. The TCGA datasets are available in the GDC portal (https:// portal.gdc.cancer.gov/).

\section{Ethics approval and consent to participate}

This study was approved by the Ethics Committee/ Institutional Review Board of the Cancer Institute (Hospital), PUMC/CAMS (No. 12-098/632). Written informed consent forms were obtained from patients for sampling and research. And all the methods in our study were carried out in accordance with the approved guidelines. All animal experiments were subject to approval by the Animal Care Committee of Shanghai Jiaotong University.

\section{Consent for publication}

Not applicable.

\section{Competing interests}

The authors declare no conflict of interest.

\section{Author details}

State Key Laboratory of Oncogenes and Related Genes, Shanghai Cancer Institute, Renji Hospital, Shanghai Jiao Tong University School of Medicine, Shanghai 200032, China. ${ }^{2}$ Institute of Nano Biomedicine and Engineering, Department of Instrument Science and Engineering, Key Laboratory for Thin Film and Microfabrication Technology of Ministry of Education, School of Electronic Information and Electronic Engineering, Shanghai Jiaotong University, Shanghai 200240, China. ${ }^{3}$ Renji Hospital, School of Medicine, Shanghai Jiao Tong University, Shanghai Institute of Digestive Disease, Shanghai, China. ${ }^{4}$ State Key Laboratory of Molecular Oncology, Center for Cancer Precision Medicine, National Cancer Center/National Clinical Research Center for Cancer/Cancer Hospital, Chinese Academy of Medical Sciences, Peking Union Medical College, Beijing 100021, China. ${ }^{5}$ Department of Medical Oncology, Fudan University Shanghai Cancer Center, Shanghai 200032, China. ${ }^{6}$ Department of Oncology, Shanghai Medical College, Fudan University, Shanghai 200032, China.

Received: 25 May 2020 Accepted: 20 August 2020

Published online: 27 August 2020

\section{References}

1. Siegel RL, Miller KD, Jemal A. Cancer statistics, 2020. CA Cancer J Clin. 2020; 70(1):7-30.

2. Herbst RS, Morgensztern D, Boshoff $C$. The biology and management of non-small cell lung cancer. Nature. 2018;553(7689):446-54.

3. Li XT, Yang JJ, Wu YL, Hou J. Toward innovative combinational immunotherapy: a systems biology perspective. Cancer Treat Rev. 2018;68: $1-8$.

4. Low JL, Walsh RJ, Ang Y, Chan G, Soo RA. The evolving immuno-oncology landscape in advanced lung cancer: first-line treatment of non-small cell lung cancer. Ther Adv Med Oncol. 2019;11:1758835919870360.

5. Goldstraw P, Chansky K, Crowley J, Rami-Porta R, Asamura H, Eberhardt WE, et al. The IASLC lung Cancer staging project: proposals for revision of the TNM stage groupings in the forthcoming (eighth) edition of the TNM classification for lung Cancer. J Thorac Oncol. 2016;11(1):39-51.
6. Hanna N, Johnson D, Temin S, Baker S Jr, Brahmer J, Ellis PM, et al. Systemic therapy for stage IV non-small-cell lung Cancer: American Society of Clinical Oncology clinical practice guideline update. J Clin Oncol. 2017;35(30):3484-515.

7. Vasconcellos VF, Marta GN, da Silva EM, Gois AF, de Castria TB, Riera R. Cisplatin versus carboplatin in combination with third-generation drugs for advanced non-small cell lung cancer. Cochrane Database Syst Rev. 2020;1: CD009256.

8. Gauthier I, Ding K, Winton T, Shepherd FA, Livingston R, Johnson DH, et al. Impact of hemoglobin levels on outcomes of adjuvant chemotherapy in resected non-small cell lung cancer: the JBR.10 trial experience. Lung Cancer. 2007;55(3):357-63.

9. Fennell DA, Summers Y, Cadranel J, Benepal T, Christoph DC, Lal R, et al. Cisplatin in the modern era: the backbone of first-line chemotherapy for non-small cell lung cancer. Cancer Treat Rev. 2016;44:42-50.

10. Lagunas VM, Melendez-Zajgla J. Nuclear factor-kappa B as a resistance factor to platinum-based Antineoplasic drugs. Met Based Drugs. 2008;2008: 576104.

11. Li Q, Yang G, Feng M, Zheng S, Cao Z, Qiu J, et al. NF-kappaB in pancreatic cancer: its key role in chemoresistance. Cancer Lett. 2018:421:127-34.

12. Ruiz EJ, Diefenbacher ME, Nelson JK, Sancho R, Pucci F, Chakraborty A, et al. LUBAC determines chemotherapy resistance in squamous cell lung cancer. J Exp Med. 2019;216(2):450-65.

13. Zhang Q, Lenardo MJ, Baltimore D. 30 years of NF-kappaB: a blossoming of relevance to human pathobiology. Cell. 2017;168(1-2):37-57.

14. Nair JS, Musi E, Schwartz GK. Selinexor (KPT-330) induces tumor suppression through nuclear sequestration of IkappaB and Downregulation of Survivin. Clin Cancer Res. 2017;23(15):4301-11.

15. Liu Q, Song J, Li H, Dong L, Dai S. Schizandrin B inhibits the cisDDPinduced apoptosis of HK2 cells by activating ERK/NFkappaB signaling to regulate the expression of survivin. Int J Mol Med. 2018;41(4):2108-16.

16. Tyagi M, Patro BS. Salinomycin reduces growth, proliferation and metastasis of cisplatin resistant breast cancer cells via NF-kB deregulation. Toxicol in Vitro. 2019;60:125-33.

17. Meylan E, Dooley AL, Feldser DM, Shen L, Turk E, Ouyang C, et al. Requirement for NF-kappaB signalling in a mouse model of lung adenocarcinoma. Nature. 2009;462(7269):104-7.

18. Basseres DS, Ebbs A, Levantini E, Baldwin AS. Requirement of the NF-kappaB subunit p65/RelA for K-Ras-induced lung tumorigenesis. Cancer Res. 2010; 70(9):3537-46.

19. Yang L, Zhou Y, Li Y, Zhou J, Wu Y, Cui Y, et al. Mutations of p53 and KRAS activate NF-kappaB to promote chemoresistance and tumorigenesis via dysregulation of cell cycle and suppression of apoptosis in lung cancer cells. Cancer Lett. 2015;357(2):520-6.

20. Xue W, Meylan E, Oliver TG, Feldser DM, Winslow MM, Bronson R, et al. Response and resistance to NF-kappaB inhibitors in mouse models of lung adenocarcinoma. Cancer Discov. 2011;1(3):236-47.

21. Liu YZ, Jiang YY, Wang BS, Hao JJ, Shang L, Zhang TT, et al. A panel of protein markers for the early detection of lung cancer with bronchial brushing specimens. Cancer Cytopathol. 2014;122(11):833-41.

22. Liu YZ, Yang H, Cao J, Jiang YY, Hao JJ, Xu X, et al. KIAA1522 is a novel prognostic biomarker in patients with non-small cell lung cancer. Sci Rep. 2016;6:24786.

23. Xie ZH, Yu J, Shang L, Zhu YQ, Hao JJ, Cai Y, et al. KIAA1522 overexpression promotes tumorigenicity and metastasis of esophageal cancer cells through potentiating the ERK activity. Onco Targets Ther. 2017;10:3743-54.

24. Li Y, Wang Y, Fan H, Zhang Z, Li N. miR-125b-5p inhibits breast cancer cell proliferation, migration and invasion by targeting KIAA1522. Biochem Biophys Res Commun. 2018;504(1):277-82.

25. Brady JJ, Chuang CH, Greenside PG, Rogers ZN, Murray CW, Caswell DR, et al. An Arnt12-driven Secretome enables lung adenocarcinoma metastatic self-sufficiency. Cancer Cell. 2016;29(5):697-710.

26. Chuang CH, Greenside PG, Rogers ZN, Brady JJ, Yang D, Ma RK, et al. Molecular definition of a metastatic lung cancer state reveals a targetable CD109-Janus kinase-stat axis. Nat Med. 2017;23(3):291-300.

27. Sanjana NE, Shalem O, Zhang F. Improved vectors and genome-wide libraries for CRISPR screening. Nat Methods. 2014;11(8):783-4.

28. Shalem O, Sanjana NE, Hartenian E, Shi X, Scott DA, Mikkelson T, et al. Genome-scale CRISPR-Cas9 knockout screening in human cells. Science. 2014;343(6166):84-7.

29. Yu G, Wang LG, Han Y, He QY. clusterProfiler: an R package for comparing biological themes among gene clusters. OMICS. 2012;16(5):284-7. 
30. Hanzelmann S, Castelo R, Guinney J. GSVA: gene set variation analysis for microarray and RNA-seq data. BMC Bioinformatics. 2013;14:7.

31. Colaprico A, Silva TC, Olsen C, Garofano L, Cava C, Garolini D, et al. TCGAbiolinks: an R/bioconductor package for integrative analysis of TCGA data. Nucleic Acids Res. 2016;44(8):e71.

32. Lambrechts D, Wauters E, Boeckx B, Aibar S, Nittner D, Burton O, et al. Phenotype molding of stromal cells in the lung tumor microenvironment. Nat Med. 2018;24(8):1277-89.

33. Butler A, Hoffman P, Smibert P, Papalexi E, Satija R. Integrating single-cell transcriptomic data across different conditions, technologies, and species. Nat Biotechnol. 2018;36(5):411-20.

34. Liu YZ, Wang BS, Jiang YY, Cao J, Hao JJ, Zhang Y, et al. MCMs expression in lung cancer: implication of prognostic significance. J Cancer. 2017;8(18): $3641-7$.

35. Buffoni L, Vavala T, Novello S. Adjuvant therapy of resected non-small cell lung Cancer: can we move forward? Curr Treat Options in Oncol. 2016; 17(10):54.

36. Isaka T, Nakayama H, Yokose T, Ito H, Katayama K, Yamada K, et al. Platinumbased adjuvant chemotherapy for stage II and stage III squamous cell carcinoma of the lung. Ann Thorac Cardiovasc Surg. 2017;23(1):19-25.

37. Liu D, Nakashima N, Nakano J, Tarumi S, Matsuura N, Nakano T, et al. Customized adjuvant chemotherapy based on biomarker examination may improve survival of patients completely resected for non-small-cell lung Cancer. Anticancer Res. 2017;37(5):2501-7.

38. Zhu CQ, Ding K, Strumpf D, Weir BA, Meyerson M, Pennell N, et al. Prognostic and predictive gene signature for adjuvant chemotherapy in resected non-small-cell lung cancer. J Clin Oncol. 2010;28(29):4417-24.

39. Platt RJ, Chen S, Zhou Y, Yim MJ, Swiech L, Kempton HR, et al. CRISPR-Cas9 knockin mice for genome editing and cancer modeling. Cell. 2014;159(2): 440-55.

40. Ben-Baruch A. Partners in crime: TNFalpha-based networks promoting cancer progression. Cancer Immunol Immunother. 2020;69(2):263-73.

41. Cabal-Hierro L, Lazo PS. Signal transduction by tumor necrosis factor receptors. Cell Signal. 2012;24(6):1297-305.

\section{Publisher's Note}

Springer Nature remains neutral with regard to jurisdictional claims in published maps and institutional affiliations.

Ready to submit your research? Choose BMC and benefit from:

- fast, convenient online submission

- thorough peer review by experienced researchers in your field

- rapid publication on acceptance

- support for research data, including large and complex data types

- gold Open Access which fosters wider collaboration and increased citations

- maximum visibility for your research: over $100 \mathrm{M}$ website views per year

At $\mathrm{BMC}$, research is always in progress.

Learn more biomedcentral.com/submissions 